\title{
Synthesis of Novel and Potent Vorapaxar Analogues
}

\author{
Emily Knight, ${ }^{a}$ Eifion Robinson, ${ }^{a}$ Natalia Smoktunowicz, ${ }^{b}$ Rachel C. Chambers, ${ }^{b}$ Abil E. Aliev, ${ }^{a}$ Graham \\ G. Inglis, ${ }^{c}$ Vijay Chudasama ${ }^{* a}$ and Stephen Caddick*a \\ Received (in $X X X, X X X)$ Xth $X X X X X X X X X 20 X X$, Accepted Xth XXXXXXXXX 20XX \\ ${ }_{5}$ DOI: 10.1039/b000000x
}

Vorapaxar is a first-in-class PAR-1 antagonistic drug based on the ent-himbacine scaffold. Detailed in this article are enantioselective and racemic routes to various novel vorapaxar analogues. Biological testing revealed these compounds to have moderate to excellent potencies against PAR-1 with the most potent analogue demonstrating an $\mathrm{IC}_{50}$ of $27 \mathrm{nM}$.

\section{${ }_{10}$ Introduction}

The recent FDA approval of vorapaxar (Figure 1) for the "reduction of thrombotic cardiovascular events in patients with a history of myocardial infarction (MI) or peripheral arterial disease (PAD)" marked an exciting development in the blockade 15 of thrombin induced cellular activities. ${ }^{1}$ Vorapaxar is a first-inclass, highly potent protease activated receptor-1 (PAR-1) antagonist. PAR-1 is a seven transmembrane G-protein coupled receptor (7TM GPCR) expressed on many cell types throughout the body including platelets, endothelial cells and fibroblasts. It is 20 agonised by an intramolecular mechanism following exposure to thrombin. ${ }^{2}$ It is an important receptor in the pathway towards thrombus formation and is associated with cardiovascular disease, as well as cancer and various fibrotic diseases. ${ }^{2}$ PAR-1 antagonists could thus be used to treat a number of serious ${ }_{25}$ conditions. With this in mind, disclosed herein is the synthesis of a series of novel vorapaxar analogues with particular focus on functionalisation at a position that has not been explored previously, i.e. the $\mathrm{C}-9$ position.
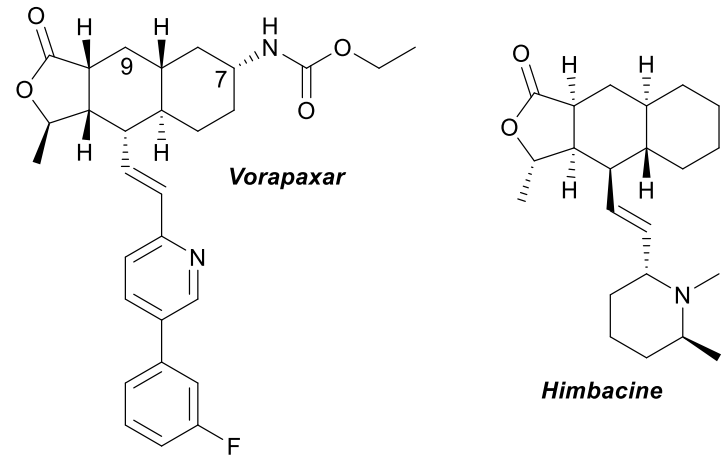

Himbacine

Fig. 1 Structures of vorapaxar and himbacine

\section{Results and Discussion}

To begin, C-9-substituted vorapaxar analogues were to be synthesised on the basis of a published partial synthetic route towards natural product himbacine (Figure 1, and Schemes 1 \& $\left.{ }_{35} 2\right)^{3-8}$ This route was designed to provide access to analogues that do not contain a carbamate group at $\mathrm{C}-7$ and have a $m-\mathrm{CF}_{3}$ on the aryl group rather a $m$-F substituent. These alterations will not affect the activity of the antagonist to any significant degree since they themselves were relatively late stage pharmacokinetic-based 40 decisions made during the development of vorapaxar that were shown to have little impact on potency, i.e. the carbamate was introduced as a means to improve oral delivery and the use of $m$ $\mathrm{F}$ in place of $m-\mathrm{CF}_{3}$ was to reduce lipophilicity. ${ }^{9}$ Moreover, the synthesis of analogues without these late stage changes, when 45 trialling a new synthetic route to vorapaxar analogues, is if anything a more sensible choice as it allows for direct comparison with the larger body of literature compounds made in the earlier stages of the development of vorapaxar. ${ }^{9}$ Alteration(s) to the analogues described herein to aid pharmacokinetic properties 50 without effecting potency can almost certainly be tuned into the core, if need be, at a later stage.

Initially, trans-methyl 2-(2-(tert-butoxy)-2oxoethyl)cyclohexanecarboxylate $(( \pm)-1)^{4}$ and 5-methyl-2(5H)furanone $(\mathbf{2})^{5}$ were synthesised using literature procedures. These 55 were then reacted together using the conditions proposed by Casey et al. to give $(3 R, 3 \mathrm{a} S, 4 R, 4 \mathrm{a} R, 8 \mathrm{a} R, 9 \mathrm{a} S)$-tert-butyl 3-methyl1,9-dioxododecahydronaphtho[2,3-c]furan-4-carboxylate (3) and $(3 R, 3 \mathrm{a} S, 4 S, 4 \mathrm{a} R, 8 \mathrm{a} R, 9 \mathrm{a} S)$-tert-butyl 3-methyl-1,9dioxododecahydronaphtho[2,3-c]furan-4-carboxylate (4) via 60 kinetic resolution, albeit in low yields (12\% and 5\% respectively) (Scheme 1). 
<smiles>CC(=O)[C@H]1CCCC[C@H]1CC(=O)OC(C)(C)C</smiles>

(士)-1

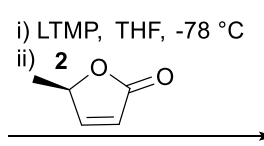

iii) KOt-Bu, -78 to $-30^{\circ} \mathrm{C}$
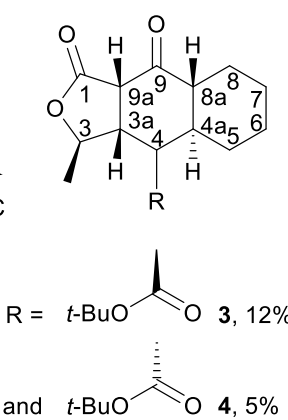

Scheme 1 Stereoselective tricycle synthesis

In order to furnish the desired C-9 functionalised vorapaxar analogues, the tert-butyl group of compounds $\mathbf{3 / 4}$ had to be 5 replaced with the biaryl motif that is essential for the PAR-1 activity of vorapaxar. ${ }^{6}$ To set about this, initially the tert-butyl group of the higher yielding tricycle (3) was removed using TFA/ $\mathrm{CH}_{2} \mathrm{Cl}_{2}$ (Scheme 2). The resulting acid was then coupled to ethanethiol using DCC and DMAP. The formed thioester (5) was 10 next reduced using mild reducing conditions of triethylsilane and catalytic palladium on carbon to give an aldehyde $(\mathbf{6}) .^{7}$ Finally, Horner-Emmons conditions were used for reaction with biaryl phosphonate ester 7, which was prepared according to a literature procedure, ${ }^{8}$ to form the desired C-9 functionalised vorapaxar 15 analogues. Notably, prior to performing the Horner-Emmons reaction, the aldehyde underwent partial epimerisation at the C-4 position. This conveniently afforded both C-4 epimers of the final C-9-keto-analogues (8 and 9, Scheme 2), and meant that the lower yielding tricycle $\mathbf{4}$ did not need to be carried through the 20 synthesis to obtain compound $\mathbf{9}$.<smiles>CC1OC(=O)[C@H]2C(=O)[C@H]3CCCC[C@H]3[C@H](C(=O)OC(C)(C)C)[C@H]12</smiles>
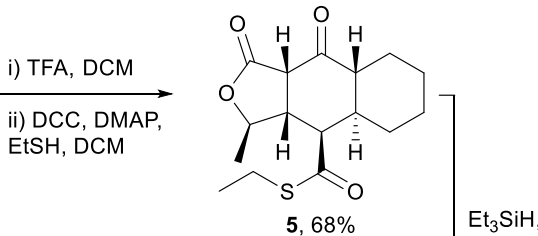
$\mathrm{Pd} / \mathrm{C}$. Acetone
9, $15 \%$

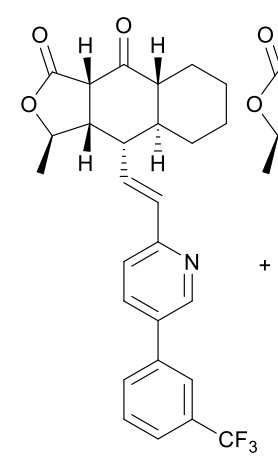

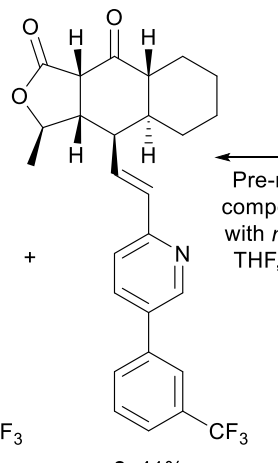

8, $41 \%$

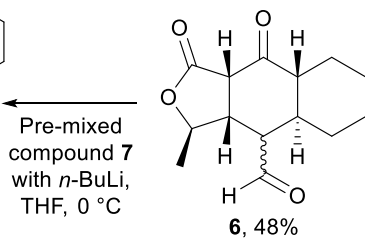

Scheme 2 Development of tricycle to give novel enantiomerically pure vorapaxar analogues $\mathbf{8}$ and $\mathbf{9}$

We noted from the outset that C-9-keto-analogues would have 25 a useful carbonyl synthetic handle to allow the exploration of SAR in a direction as yet unexplored on vorapaxar. To the best of our knowledge, there are no vorapaxar analogues with substitution at the C-9 position. As an initial foray into the novel
SAR possibilities, the ketone of compound $\mathbf{8}$ was reduced to an 30 hydroxyl group using $\mathrm{NaBH}_{4}$ to give compounds $\mathbf{1 0}$ and $\mathbf{1 1}$ (Figure 2) in $38 \%$ and $29 \%$ yield respectively. This was a significant transformation as it returned sp3 hybridisation at C-9, making the tricycle conformation more similar to that of vorapaxar. Additionally, compounds 8-11 would prove useful in 35 exploring the effect on activity of $\mathrm{sp} 2$ vs. $\mathrm{sp} 3$ hybridisation at C-9. The stereochemical assignment of compounds 8-11 was made on the basis of ${ }^{1} \mathrm{H}$ NMR coupling constants and 2D NMR analysis; see ESI for further details.
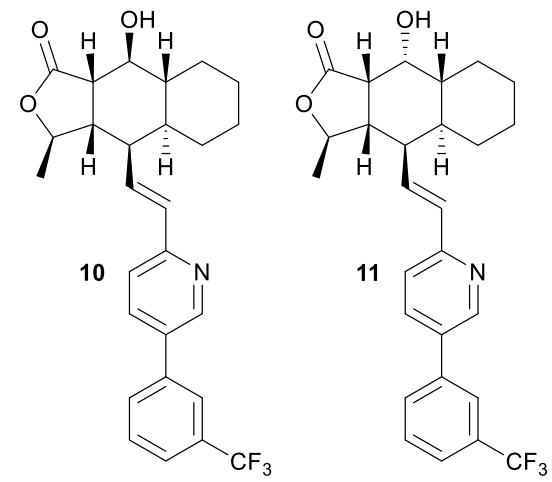

$40 \quad$ Fig. 2 Novel enantiomerically pure vorapaxar analogues $\mathbf{1 0}$ and $\mathbf{1 1}$

Next we used commercially available $2-(5 H)$-furanone in place of methyl-furanone 2 for reaction with trans-methyl 2-(2-(tertbutoxy)-2-oxoethyl)cyclohexanecarboxylate ( \pm )-1 as a conduit to gain access to further distinct analogues of vorapaxar in terms of 45 relative ring junction stereochemistry. The absence of the methyl group on 2-(5H)-furanone, and thus in subsequent vorapaxar analogues, would have no bearing as, in line with several examples in the literature, this methyl group is not thought to have an effect on PAR-1 antagonism. ${ }^{10}$ The reaction of ( \pm )-1 and ${ }_{50} 2$ - $(5 H)$-furanone led to the formation of racemic compounds $( \pm)-12$ and $( \pm)-13$, which to our delight had distinct relative stereochemistries at C-9a/C-3a compared with $\mathrm{C}-8 \mathrm{a} / \mathrm{C}-4 \mathrm{a}$. For this reaction, the addition of $\mathrm{KO} t-\mathrm{Bu}$ was not required for tricycle synthesis, which may have contributed to an increased overall 55 yield of $51 \%$ for tricycle formation.

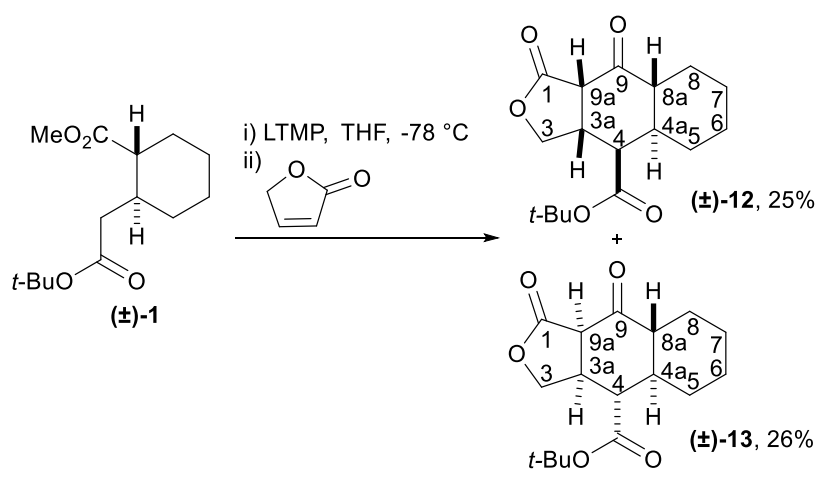

Scheme 3 Racemic tricycle synthesis

The synthetic route detailed in Scheme 2 was next repeated on $( \pm)-12$ and $( \pm)-13$, which led to the formation of racemic PAR-1 60 analogues ( \pm )-14-16. The racemic compounds were easier to synthesise and use of ( \pm )-12 and ( \pm )-13 allowed access to PAR-1 analogues with tricycle scaffolds with differing relative 
stereochemical assignment at C-9a/C-3a compared with $\mathrm{C}-8 \mathrm{a} / \mathrm{C}$ $4 \mathrm{a}$, i.e. $( \pm)-\mathbf{1 4} / 15$ and $( \pm)-16$, respectively. The enantiomeric pairs were then separated out into $(+)$ and $(-)$ enantiomers by chiral chromatography as the absolute stereochemistry of vorapaxar is 5 believed to be important for PAR-1 potency. ${ }^{11}$
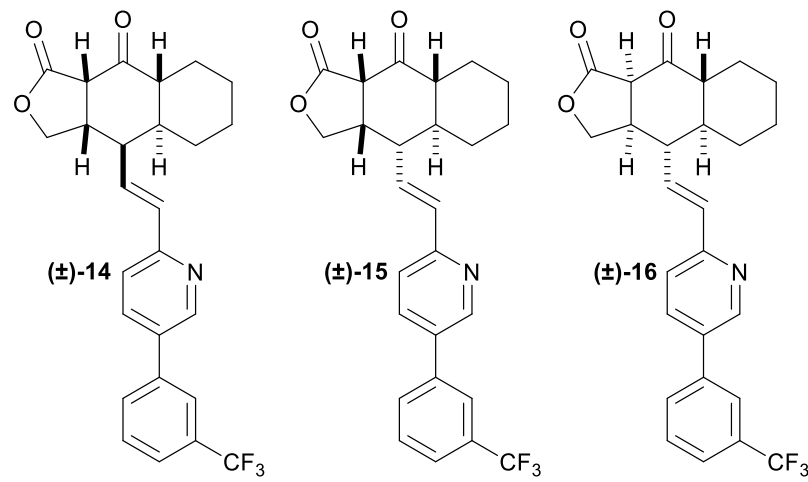

Fig. 3 Racemic novel vorapaxar analogues ( \pm )-14-16

The results of the synthetic efforts gave 10 key compounds which were tested in a biological assay to determine their potency 10 against PAR-1 (Figures 2-5, Table 1). The assay was based on monitoring PAR-1 induced calcium flux in response to thrombin using FLIPR. ${ }^{12}$ The novel analogues showed concentrationdependent antagonism in human lung fibroblasts vs. $10 \mathrm{nM}$ thrombin as the agonist. The academic tool, RWJ-58259, ${ }^{13}$ was 15 used as a positive control and for a potency comparator.

Enantiopure compounds 8-11 were tested initially (Figure 4). The most potent of these analogues, compound $\mathbf{9}$, gave an $\mathrm{IC}_{50}$ of $0.5 \mu \mathrm{M}$. This meant that it had a similar potency to that of RWJ$58259\left(\mathrm{IC}_{50}=0.17 \mu \mathrm{M}\right)$. Unfortunately, the potencies of the 20 compounds did not quite match that of vorapaxar $\left(\mathrm{IC}_{50}=13 \mathrm{nM}\right.$, in this assay). It was interesting to note that the hydroxylanalogues $\left(\mathrm{IC}_{50}: \mathbf{1 0}=72 \mu \mathrm{M}\right.$ and $\left.\mathbf{1 1}=120 \mu \mathrm{M}\right)$ were less potent than the keto-analogues $\left(\mathrm{IC}_{50}: \mathbf{8}=10.5 \mu \mathrm{M}\right.$ and $\mathbf{9}=0.5 \mu \mathrm{M}$ ), indicating that the $\mathrm{sp} 2$ vs. $\mathrm{sp} 3$ hybridisation at C-9 was not critical 25 to activity, and that (if anything) sp2 hybridisation may be moderately preferred.

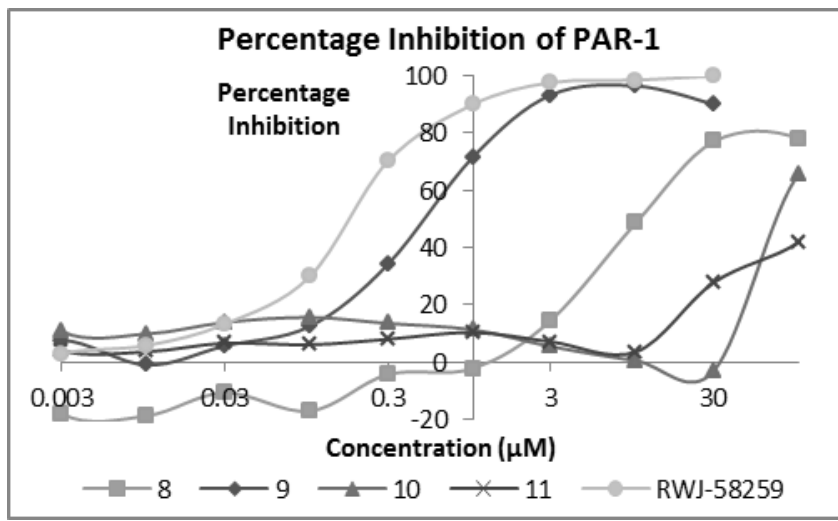

Fig. 4 The average $(n=6)$ percentage inhibition observed upon the addition of thrombin $(10 \mathrm{nM})$ to human lung fibroblasts in buffer preincubated with Fluo-4 NW dye mix and $0.003-300 \mu \mathrm{M}$ antagonist; Compounds 8, 9, 10, 11 and RWJ-58259

Next, each of the enantiopure compounds obtained from ( \pm )14-15, were tested. In all cases, the compounds failed to reach
$100 \%$ inhibition at a concentration of $30 \mu \mathrm{M}$. The concentration 35 range was not extended to $300 \mu \mathrm{M}$, as seen with the compounds 8-11, because solubility issues were starting to be observed at 30 $\mu \mathrm{M}$. As seen in the vorapaxar literature, one enantiomer was shown to be more potent than its pair $\left(\mathrm{IC}_{50}:(-)-\mathbf{1 4}=2.4 \mu \mathrm{M},(+)\right.$ $14=15 \mu \mathrm{M},(-)-\mathbf{1 5}=0.1 \mu \mathrm{M}$ and $(+)-\mathbf{1 5}=7.5 \mu \mathrm{M}) .{ }^{11}$ In this 40 series, the most potent compound (i.e. (-)-15) was slightly more potent than both compound 9 and RWJ-58259.

Finally, the analogues with distinct tricycle ring juncture stereochemistry, i.e. (+)-16 and (-)-16, were tested (Figure 5). The enantiomeric pair derived from compound $\mathbf{1 6}$ had vastly ${ }_{45}$ distinct potencies (Figure 5). Compound (-)-16 with an $\mathrm{IC}_{50}$ of 27 $\mathrm{nM}$ was the most potent of all of the compounds tested. It was more potent than $\mathrm{RWJ}-58259\left(\mathrm{IC}_{50}=170 \mathrm{nM}\right.$, in this assay) and approached the potency of vorapaxar $\left(\mathrm{IC}_{50}=13 \mathrm{nM}\right)$. Conversely, its enantiomeric partner ((+)-16) gave a ca. 100-fold increase in ${ }_{50} \mathrm{IC}_{50}$. This is one of the lowest potencies of the tested ketoanalogues and 1000 times less potent than vorapaxar.t.

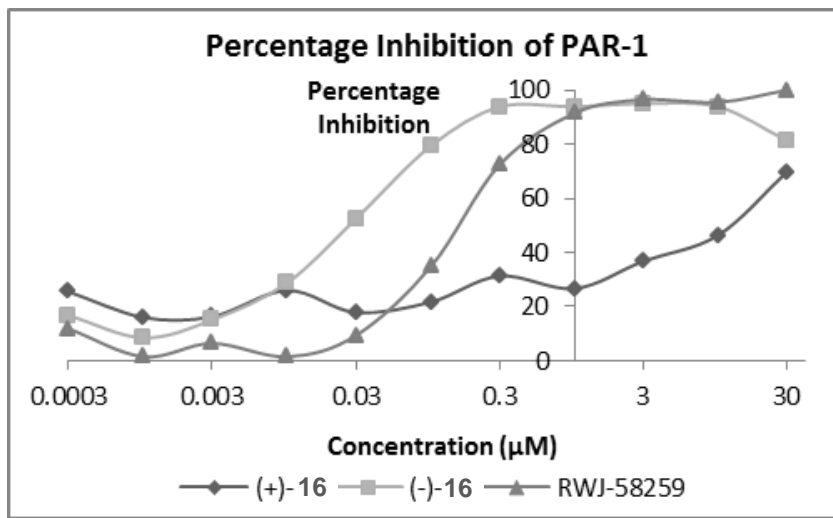

Fig. 5 The average $(n=6)$ percentage inhibition observed upon the addition of thrombin ( $10 \mathrm{nM}$ ) to human lung fibroblasts in buffer pre55 incubated with Fluo-4 NW dye mix and $0.0003-30 \mu \mathrm{M}$ antagonist; Compounds (+)-16, (-)-16 and RWJ-58259

Table $1 \mathrm{IC}_{50}$ values of vorapaxar, all vorapaxar analogues and RWJ58259

$\begin{array}{cc}\text { Compound } & \mathbf{I C}_{\mathbf{5 0}}(\boldsymbol{\mu M})^{a} \\ \text { Vorapaxar } & 0.013 \\ \mathbf{8} & 10.5 \\ \mathbf{9} & 0.5 \\ \mathbf{1 0} & 72 \\ \mathbf{1 1} & 120 \\ \mathbf{( + ) - 1 4} & 15 \\ \mathbf{( - ) - 1 4} & 2.4 \\ \mathbf{( + ) - 1 5} & 7.5 \\ \mathbf{( - ) - 1 5} & 0.1 \\ \mathbf{( + ) - 1 6} & 13 \\ \mathbf{( - ) - 1 6} & 0.027 \\ \text { RWJ-58259 } & 0.17\end{array}$

${ }^{a}$ See ESI for details on biological assay used to determine these values.

\section{${ }_{60}$ Conclusions}

In conclusion, an enantioselective route to himbacine-like tricycles has been adapted and developed to give a number of novel vorapaxar analogues which were tested biologically. The nanomolar potency found with compound (-)-16 is very 65 significant because the novel scaffold has the potential for further growth to investigate the SAR of vorapaxar, a first-in-class 
PAR-1 antagonist drug. Whilst there is excellent recent work on C-7-spirocyclic analogues of vorapaxar, ${ }^{14}$ and examples of functionalisation at other positions on the core, to the best of our knowledge these are the first PAR-1 active vorapaxar analogues 5 with substitution at the $\mathrm{C}-9$ position or with the differing relative stereochemistry on the tricycle. Consequently, especially with readily functionalisable groups at the $\mathrm{C}-9$ position, there is the prospect of developing further novel and potent PAR-1 inhibitors which could be used for the treatment of cancer and various 10 fibrotic diseases.

\section{Experimental section}

\section{General Experimental}

All solvents employed in this study were reagent grade. All reagents were purchased from Sigma-Aldrich, UK and Alfa 15 Aesar, UK and used as received unless otherwise stated. All reactions were magnetically stirred and monitored by thin layer chromatography (TLC) on pre-coated silica gel plates $(254 \mu \mathrm{m})$ and/or by LCMS. Silica plates were initially examined under UV light and then developed using aqueous basic potassium 20 permanganate stain. LCMS analysis was conducted on either System A, an Acquity UPLC BEH C18 column $(2.1 \mathrm{~mm} \times 50$ $\mathrm{mm}$ ID, $1.7 \mu \mathrm{m}$ packing diameter) eluting with $0.1 \%$ formic acid in $\mathrm{H}_{2} \mathrm{O}$ (solvent A) and $0.1 \%$ formic acid in acetonitrile (MeCN) (solvent $\mathrm{B}$ ), using the following elution gradient 0.0-1.5 min $253-100 \% \mathrm{~B}, 1.5-1.9 \mathrm{~min} 100 \% \mathrm{~B}, 1.9-2.0 \mathrm{~min} 3 \% \mathrm{~B}$, at a flow rate of $1 \mathrm{~mL}$ min-1 at $40{ }^{\circ} \mathrm{C}$. The UV detection was an averaged signal from wavelength of 210 to $350 \mathrm{~nm}$, and mass spectra were recorded on a mass spectrometer using alternate-scan electrospray positive and negative mode ionization (ES +ve and ES -ve); or 30 System B, an Acquity UPLC BEH C18 column $(50 \mathrm{~mm} \times 2.1$ $\mathrm{mm}$ ID, $1.7 \mu \mathrm{m}$ packing diameter) eluting with $10 \mathrm{mM}$ ammonium bicarbonate $\left(\left(\mathrm{NH}_{4}\right) \mathrm{HCO}_{3}\right)$ in $\mathrm{H}_{2} \mathrm{O}$ adjusted to $\mathrm{pH} 10$ with ammonia solution (solvent $\mathrm{A}$ ) and $\mathrm{MeCN}$ (solvent $\mathrm{B}$ ) using the following elution gradient $0-1.5 \mathrm{~min} 1-97 \% \mathrm{~B}, 1.5-1.9 \mathrm{~min}$ ${ }_{35} 97 \% \mathrm{~B}, 1.9-2.0 \mathrm{~min} 100 \% \mathrm{~B}$ at a flow rate of $1 \mathrm{~mL} \mathrm{~min}-1$ at 40 ${ }^{\circ} \mathrm{C}$. Preperative TLC was carried out using $20 \mathrm{~cm}$ x $20 \mathrm{~cm}$ glass TLC plates with a silica gel 60 matrix, supplied by EMD/Merck KGaA. Flash chromatography was carried out with silica gel (33$70 \mu \mathrm{m})$ supplied by Merck Co.. Automated column 40 chromatography was performed using pre-packed silica gel columns on a Flashmaster II. The Flashmaster II is an automated multiuser flash chromatography system, available from Argonaut Technologies Ltd, which utilizes disposable, normal phase, SPE cartridges $(2-100 \mathrm{~g})$. Chiral column chromatography was 45 performed using various columns and conditions, see below for details. Quoted yields refer to chromatographically and spectroscopically pure compounds unless otherwise stated. ${ }^{1} \mathrm{H}$ NMR spectra were recorded at $600 \mathrm{MHz}$ with a Bruker AMX600. ${ }^{13} \mathrm{C}$ NMR spectra were recorded at $150 \mathrm{MHz}$. Chemical shifts $(\delta$ 50 values) are reported in parts per million ( $\mathrm{ppm}$ ) whilst coupling constants are reported in Hertz $(\mathrm{Hz})$.

\section{trans-Methyl}

oxoethyl)cyclohexanecarboxylate (1)

${ }_{55} n$-BuLi (1.6 M in hexanes) $(70.0 \mathrm{~mL}, 112 \mathrm{mmol})$ was added to a stirred solution of DIPA $(15.7 \mathrm{~mL}, 112 \mathrm{mmol})$ in anhydrous THF
(30 $\mathrm{mL}$ ) at $-78{ }^{\circ} \mathrm{C}$, under Ar. The solution was stirred at $-78{ }^{\circ} \mathrm{C}$ for 20 mins. A solution of tert-butyl acetate $(15.0 \mathrm{~mL}, 112 \mathrm{mmol})$ was added and stirring continued for $30 \mathrm{~min}$ at $-78{ }^{\circ} \mathrm{C}$. Next, a 60 solution of $(E)$-methyl 7-iodohept-2-enoate $(13.7 \mathrm{~g}, 51 \mathrm{mmol})$ in anhydrous THF $(15 \mathrm{~mL})$ was added. The reaction mixture was stirred for a further $30 \mathrm{~min}$ at $-78{ }^{\circ} \mathrm{C}$. Finally, solid $\mathrm{KO} t$-Bu $(12.6$ $\mathrm{g}, 112 \mathrm{mmol}$ ) was added and stirring continued for a further $1 \mathrm{~h}$ at $-78{ }^{\circ} \mathrm{C}$. The reaction mixture was quenched with sat. aq. $\mathrm{NH}_{4} \mathrm{Cl}$ $65(100 \mathrm{~mL})$ and diluted with $\mathrm{H}_{2} \mathrm{O}(50 \mathrm{~mL})$. An extraction into EtOAc $(2 \times 100 \mathrm{~mL})$ was completed and the combined organic layers were dried (MgSO4), filtered and concentrated in vacuo to yield a crude pale yellow liquid. Multiple purifications by flash column chromatography $\left(0-2 \% \mathrm{Et}_{2} \mathrm{O} / \mathrm{CH}_{2} \mathrm{Cl}_{2}\right),\left(0-10 \% \mathrm{Et}_{2} \mathrm{O} / \mathrm{Pet}\right.$. 70 Ether) and (0-2\% $\mathrm{Et}_{2} \mathrm{O} /$ toluene) yielded trans-methyl 2-(2-(tertbutoxy)-2-oxoethyl)cyclohexanecarboxylate (1) (3.1 g, $12 \mathrm{mmol}$, $24 \%$ ) as a colourless liquid; bp. $110-115{ }^{\circ} \mathrm{C}$ (at $2.1 \mathrm{mbar}$ ); ${ }^{1} \mathrm{H}$ NMR (600 MHz, $\left.\mathrm{CDCl}_{3}\right) \delta 3.66\left(3 \mathrm{H}, \mathrm{s}, \mathrm{COOCH}_{3}\right), 2.26(1 \mathrm{H}, \mathrm{d}, J$ $\left.=10.9, \mathrm{CHCOO}^{\mathrm{t}} \mathrm{Bu}\right), 2.12\left(1 \mathrm{H}, \mathrm{dt}, J=11.2,3.6, \mathrm{C}^{2} H\right), 2.05-$ $751.98\left(2 \mathrm{H}, \mathrm{m}, \mathrm{C}^{1} \mathrm{H} \& \mathrm{CH}_{\mathrm{HCOO}}^{\mathrm{t}} \mathrm{Bu}\right), 1.89(1 \mathrm{H}, \mathrm{qd}, J=1.9,13.2$, $\left.\mathrm{C}^{3} H \mathrm{H}\right), 1.84\left(1 \mathrm{H}, \mathrm{dd}, J=2.1,13.0, \mathrm{C}^{6} H \mathrm{H}\right), 1.74(1 \mathrm{H}, \mathrm{dtd}, J=$ $\left.12.8,3.4,1.1, \mathrm{C}^{4} H \mathrm{H}\right), 1.72-1.68\left(1 \mathrm{H}, \mathrm{m}, \mathrm{C}^{5} H \mathrm{H}\right), 1.47(1 \mathrm{H}, \mathrm{td}, J=$ 12.4, 2.6, $\left.\mathrm{C}^{3} \mathrm{H} H\right), 1.43\left(9 \mathrm{H}, \mathrm{s}, \mathrm{C}\left(\mathrm{CH}_{3}\right)_{3}\right), 1.30(1 \mathrm{H}, \mathrm{tq}, J=12.8$, $\left.3.4, \mathrm{C}^{5} \mathrm{H} H\right), 1.21\left(1 \mathrm{H}, \mathrm{tq}, J=13.2,3.8, \mathrm{C}^{4} \mathrm{H} H\right), 1.04(1 \mathrm{H}, \mathrm{dq}, J=$ $\left.{ }_{80} 13.2, \quad 3.4, \mathrm{C}^{6} \mathrm{H} H\right) ;{ }^{13} \mathrm{C} \mathrm{NMR}\left(150 \mathrm{MHz}, \mathrm{CDCl}_{3}\right) \delta 176.1$ (COCOMe), $171.8\left(C \mathrm{COCO}^{\mathrm{t}} \mathrm{Bu}\right), 80.4\left(\mathrm{C}\left(\mathrm{CH}_{3}\right)_{3}\right), 51.7\left(\mathrm{CH}_{3}\right), 49.0$ $\left(C^{2} \mathrm{H}\right), 40.9\left(C \mathrm{H}_{2} \mathrm{COO}^{\mathrm{t}} \mathrm{Bu}\right), 36.3\left(C^{l} \mathrm{H}\right), 31.3\left(C^{6} \mathrm{H}\right), 30.0\left(C^{3} \mathrm{H}\right)$, $28.2\left(\mathrm{C}\left(\mathrm{CH}_{3}\right)_{3}\right), 25.5\left(\mathrm{C}^{5} \mathrm{H}\right), 25.4\left(\mathrm{C}^{4} \mathrm{H}\right)$; IR (thin film) $2982(\mathrm{C}-$ H), 2930 (C-H), $2854(\mathrm{C}-\mathrm{H}), 1713(\mathrm{C}=\mathrm{O}), 1451(\mathrm{C}-\mathrm{H}), 1366(\mathrm{C}-$ $\left.{ }_{85} \mathrm{O}\right), 1247$ (C-O), 1151, 1108, 1025, $845 \mathrm{~cm}^{-1} ; \mathrm{m} / \mathrm{z}(\mathrm{ES}+) 257$ $\left(100 \%,[\mathrm{M}+\mathrm{H}]^{+}\right)$; HRMS $(\mathrm{CI})$ calcd for $\mathrm{C}_{13} \mathrm{H}_{11} \mathrm{O}_{3}$ [M-OMe] 225.14907, observed 225.14872.

tert-Butyl 3-methyl-1,9-dioxododecahydronaphtho[2,390 c]furan-4-carboxylate (3 and 4)

$n$-BuLi (2.5 M in hexane) (3.60 ml, $9.00 \mathrm{mmol})$ was added dropwise to a stirred solution of freshly distilled TMP $(1.54 \mathrm{~mL}$, $9.00 \mathrm{mmol})$ in anhydrous THF $(10 \mathrm{~mL})$ at $-78{ }^{\circ} \mathrm{C}$, under Ar. The solution was warmed to $0{ }^{\circ} \mathrm{C}$ and then re-cooled to $-78{ }^{\circ} \mathrm{C}$. A 95 solution of trans-methyl 2-(2-(tert-butoxy)-2oxoethyl)cyclohexanecarboxylate (1) $(1.10 \mathrm{~g}, 4.30 \mathrm{mmol})$ in anhydrous THF $(5 \mathrm{~mL})$ was added, dropwise, and stirring continued for $45 \mathrm{~min}$ at $-78{ }^{\circ} \mathrm{C}$. Next, a solution of 5-methyl2(5H)-furanone (2) (294 mg, $0.30 \mathrm{mmol})$ in anhydrous THF (5 $100 \mathrm{~mL}$ ) was added, dropwise, and stirring continued for $1 \mathrm{~h}$ at -78 ${ }^{\circ} \mathrm{C}$. Finally, $\mathrm{KO} t$-Bu $(1.00 \mathrm{~g}, 9.00 \mathrm{mmol})$ was added. The reaction mixture was warmed to $-40{ }^{\circ} \mathrm{C}$, stirred for a further $3 \mathrm{~h}$ and then quenched with sat. aq. $\mathrm{NH}_{4} \mathrm{Cl}(20 \mathrm{~mL})$. An extraction into EtOAc $(2 \mathrm{x} 40 \mathrm{~mL})$ was done and the combined organic layers were 105 dried $\left(\mathrm{MgSO}_{4}\right)$, filtered and concentrated in vacuo to yield a crude yellow oily solid. Multiple purifications by flash column chromatography yielded $(3 R, 3 \mathrm{a} S, 4 R, 4 \mathrm{a} R, 8 \mathrm{a} R, 9 \mathrm{a} S)$-tert-butyl 3methyl-1,9-dioxododecahydronaphtho[2,3-c]furan-4-carboxylate (3) $(117 \mathrm{mg}, 0.36 \mathrm{mmol}, 12 \%)$ and $(3 R, 3 \mathrm{aS}, 4 S, 4 \mathrm{a} R, 8 \mathrm{a} R, 9 \mathrm{a} S)$-tert110 butyl 3-methyl-1,9-dioxododecahydronaphtho[2,3-c]furan-4carboxylate (4) $(47.5 \mathrm{mg}, 0.15 \mathrm{mmol}, 5 \%)$ as white solids. Data for $\quad(3 R, 3 \mathrm{a} S, 4 R, 4 \mathrm{a} R, 8 \mathrm{a} R, 9 \mathrm{a} S)$-tert-butyl 3-methyl-1,9dioxododecahydronaphtho[2,3-c]furan-4-carboxylate $\quad(3):{ }^{1} \mathrm{H}$ NMR $\left(600 \mathrm{MHz}, \mathrm{CDCl}_{3}\right) \delta 4.24\left(1 \mathrm{H}, \mathrm{dd}, J=10.2,6.0, \mathrm{C}^{3} \mathrm{HMe}\right)$, 
$3.77\left(1 \mathrm{H}, \mathrm{d}, J=8.3, \mathrm{C}^{9 \mathrm{a}} H\right), 2.71-2.66\left(1 \mathrm{H}, \mathrm{m}, \mathrm{C}^{8 \mathrm{a}} H\right), 2.51(1 \mathrm{H}, \mathrm{d}$, $\left.J=4.9, \mathrm{C}^{4} H\right), 2.01-1.96\left(1 \mathrm{H}, \mathrm{m}, \mathrm{C}^{8} H \mathrm{H}\right), 1.84-1.72\left(4 \mathrm{H}, \mathrm{m}, \mathrm{C}^{4 \mathrm{a}} H\right.$, $\left.\mathrm{C}^{5} \mathrm{HH}, \mathrm{C}^{6} \mathrm{HH} \& \mathrm{C}^{7} \mathrm{HH}\right), 1.50\left(9 \mathrm{H}, \mathrm{s}, \mathrm{C}\left(\mathrm{CH}_{3}\right)_{3}\right), 1.42(3 \mathrm{H}, \mathrm{d}, J=$ 6.0, $\left.\mathrm{C}^{3} \mathrm{HCH}_{3}\right), 1.33-1.23\left(2 \mathrm{H}, \mathrm{m}, \mathrm{C}^{5} \mathrm{H} H \& \mathrm{C}^{8} \mathrm{H} H\right), 1.22-1.14$ ${ }_{5}\left(2 \mathrm{H}, \mathrm{m}, \mathrm{C}^{6} \mathrm{H} H \& \mathrm{C}^{7} \mathrm{H} H\right) ;{ }^{13} \mathrm{C}$ NMR $\left(150 \mathrm{MHz}, \mathrm{CDCl}_{3}\right) \delta 204.0$ $\left(C^{9} \mathrm{O}\right), 172.0\left(C^{1} \mathrm{OO}^{t} \mathrm{Bu}\right), 171.1\left(C^{l} \mathrm{O}\right), 82.5\left(C\left(\mathrm{CH}_{3}\right)_{3}\right), 77.6$ $\left(C^{3} \mathrm{HMe}\right), 54.5\left(C^{9 a} \mathrm{H}\right), 48.4\left(C^{3 a} \mathrm{H}\right), 48.1\left(C^{8 a} \mathrm{H}\right), 44.1\left(C^{4} \mathrm{H}\right)$, $41.0\left(C^{A a} \mathrm{H}\right), 31.4\left(C^{5} \mathrm{H}_{2}\right), 28.3\left(\mathrm{C}\left(C_{3}\right)_{3}\right), 25.7\left(C^{8} \mathrm{H}_{2}\right), 25.5$ $\left(C^{6} \mathrm{H}_{2}\right), 25.0\left(C^{7} \mathrm{H}_{2}\right), 19.1\left(\mathrm{C}^{3} \mathrm{HCH}_{3}\right)$; IR (thin film) $2988(\mathrm{C}-\mathrm{H})$, $102918(\mathrm{C}-\mathrm{H}), 2855(\mathrm{C}-\mathrm{H}), 1790(\mathrm{C}=\mathrm{O}), 1727(\mathrm{C}=\mathrm{O}), 1705(\mathrm{C}=\mathrm{O})$, 1198 (C-O), 1197, 1133, 1061, 913, $835 \mathrm{~cm}^{-1} ; \mathrm{m} / \mathrm{z}$ (CI) 267 $\left(100 \%\right.$, $\left.\left[\mathrm{M}-\mathrm{C}_{4} \mathrm{H}_{9}\right]^{+}\right)$; HRMS (CI) calcd for $\mathrm{C}_{18} \mathrm{H}_{27} \mathrm{O}_{5}[\mathrm{M}+\mathrm{H}]^{+}$ 323.1853, observed 323.1855. Data for $(3 R, 3 \mathrm{a} S, 4 S, 4 \mathrm{a} R, 8 \mathrm{a} R, 9 \mathrm{a} S)$ tert-butyl 3-methyl-1,9-dioxododecahydronaphtho[2,3-c]furan-415 carboxylate (4): ${ }^{1} \mathrm{H}$ NMR $\left(600 \mathrm{MHz}, \mathrm{CDCl}_{3}\right) \delta 4.54(1 \mathrm{H}, \mathrm{dq}, J=$ $\left.10.2,6.0, \mathrm{C}^{3} H \mathrm{Me}\right), 3.48\left(1 \mathrm{H}, \mathrm{d}, J=8.7, \mathrm{C}^{9 \mathrm{a}} H\right), 2.94(1 \mathrm{H}, \mathrm{ddd}, J=$ $\left.10.2,8.7,5.3, \mathrm{C}^{3 \mathrm{a}} H\right), 2.81\left(1 \mathrm{H}, \mathrm{dd}, J=11.5,5.3, \mathrm{C}^{4} H\right), 2.08(1 \mathrm{H}$, $\left.\mathrm{td}, J=11.8,3.2, \mathrm{C}^{8 \mathrm{a}} H\right), 1.96-1.88\left(3 \mathrm{H}, \mathrm{m}, \mathrm{C}^{4 \mathrm{a}} H, \mathrm{C}^{5} H \mathrm{H} \& \mathrm{C}^{8} H \mathrm{H}\right)$, 1.84-1.80 (1H, m, C $\left.\mathrm{C}^{6} \mathrm{HH}\right), 1.75-1.71\left(1 \mathrm{H}, \mathrm{m}, \mathrm{C}^{7} \mathrm{HH}\right), 1.48(9 \mathrm{H}, \mathrm{s}$, $\left.{ }_{20} \mathrm{C}\left(\mathrm{CH}_{3}\right)_{3}\right), 1.36\left(3 \mathrm{H}, \mathrm{d}, J=6.0, \mathrm{C}^{3} \mathrm{HCH}_{3}\right), 1.39-1.34(1 \mathrm{H}, \mathrm{m}$, $\left.\mathrm{C}^{8} \mathrm{H} H\right), 1.26-1.17\left(2 \mathrm{H}, \mathrm{m}, \mathrm{C}^{6} \mathrm{H} H \& \mathrm{C}^{7} \mathrm{H} H\right), 1.13-1.05(1 \mathrm{H}, \mathrm{m}$, $\left.\mathrm{C}^{5} \mathrm{H} H\right) ;{ }^{13} \mathrm{C} \mathrm{NMR}\left(150 \mathrm{MHz}, \mathrm{CDCl}_{3}\right) \delta 203.0\left(C^{9} \mathrm{O}\right), 171.6$ $\left(C \mathrm{OO}^{\mathrm{t}} \mathrm{Bu}\right), 170.2\left(C^{l} \mathrm{O}\right), 82.5\left(C\left(\mathrm{CH}_{3}\right)_{3}\right), 77.3\left(C^{3} \mathrm{HMe}\right), 56.3$ $\left(C^{9 a} \mathrm{H}\right), 52.4\left(C^{8 a} \mathrm{H}\right), 48.0\left(C^{3 a} \mathrm{H} \& C^{4} \mathrm{H}\right), 41.5\left(C^{4 a} \mathrm{H}\right), 32.0$ ${ }_{25}\left(C^{5} \mathrm{H}_{2}\right), 28.3\left(\mathrm{C}\left(\mathrm{CH}_{3}\right)_{3}\right), 25.1\left(C^{8} \mathrm{H}_{2}\right), 24.9\left(C^{6} \mathrm{H}_{2}\right), 24.8\left(C^{7} \mathrm{H}_{2}\right)$, $20.3\left(\mathrm{C}^{3} \mathrm{HCH}_{3}\right)$; IR (thin film) $2978(\mathrm{C}-\mathrm{H}), 2933(\mathrm{C}-\mathrm{H}), 2857(\mathrm{C}-$ $\mathrm{H}), 1784(\mathrm{C}=\mathrm{O}), 1717(\mathrm{C}=\mathrm{O})$, 1367, $1194(\mathrm{C}-\mathrm{O})$, 1146, 1066, 985, $751 \mathrm{~cm}^{-1} ; \mathrm{m} / \mathrm{z}$ (CI) $267\left(100 \%,\left[\mathrm{M}-\mathrm{C}_{4} \mathrm{H}_{9}\right]^{+}\right)$; HRMS (CI) calcd for $\mathrm{C}_{18} \mathrm{H}_{27} \mathrm{O}_{5}[\mathrm{M}+\mathrm{H}]^{+}$323.1853, observed 323.1856.

30

$(3 R, 3 \mathrm{a} S, 4 R, 4 \mathrm{a} R, 8 \mathrm{a} R, 9 \mathrm{a} S)$-3-Methyl-1,9-

dioxododecahydronaphtho[2,3-c]furan-4-carboxylic acid

TFA $(0.5 \mathrm{~mL})$ was added dropwise to a stirred solution of (3R,3aS, 4R,4aR,8aR,9aS)-tert-butyl 3-methyl-1,9-

35 dioxododecahydronaphtho[2,3-c]furan-4-carboxylate $(86.0 \mathrm{mg}$, $0.27 \mathrm{mmol})$ in $\mathrm{CH}_{2} \mathrm{Cl}_{2}(0.5 \mathrm{~mL})$ at $0{ }^{\circ} \mathrm{C}$, under Ar. The reaction mixture was stirred at $20^{\circ} \mathrm{C}$ for $16 \mathrm{~h}$ before being concentrated in vacuo. The residue was azeotroped with toluene to yield $(3 R, 3 \mathrm{a} S, 4 R, 4 \mathrm{a} R, 8 \mathrm{a} R, 9 \mathrm{a} S)$-3-methyl-1,9-

40 dioxododecahydronaphtho[2,3-c]furan-4-carboxylic acid $(69.8$ $\mathrm{mg}, 0.26 \mathrm{mmol}, 99 \%)$ as a white solid; ${ }^{1} \mathrm{H}$ NMR $(600 \mathrm{MHz}$, $\left.\mathrm{MeOH}-\mathrm{d}_{4}\right) \delta 4.39\left(1 \mathrm{H}, \mathrm{td}, J=11.7,6, \mathrm{C}^{3} H \mathrm{Me}\right), 3.82(1 \mathrm{H}, \mathrm{d}, J=$ 8.7, $\left.\mathrm{C}^{9 \mathrm{a}} H\right), 2.87\left(1 \mathrm{H}, \mathrm{dd}, J=10.4,8.7, \mathrm{C}^{3 \mathrm{a}} H\right), 2.76(1 \mathrm{H}, \mathrm{td}, J=$ 11.7, 3.4, $\left.\mathrm{C}^{8 \mathrm{a}} H\right), 2.71\left(1 \mathrm{H}, \mathrm{d}, J=4.9, \mathrm{C}^{4} H\right), 1.97(1 \mathrm{H}$, dddd, $J=$ $\left.4512.4,11.7,4.9,3.8, \mathrm{C}^{4 \mathrm{a}} H\right), 1.96-1.92\left(1 \mathrm{H}, \mathrm{m}, \mathrm{C}^{8} H \mathrm{H}\right), 1.87-1.82$ $\left(1 \mathrm{H}, \mathrm{m}, \mathrm{C}^{5} H \mathrm{H}\right), 1.81-1.74\left(2 \mathrm{H}, \mathrm{m}, \mathrm{C}^{6} H \mathrm{H} \& \mathrm{C}^{7} H \mathrm{H}\right), 1.42(3 \mathrm{H}, \mathrm{d}$, $\left.J=6.0, \mathrm{C}^{3} \mathrm{HCH}_{3}\right), 1.40-1.36\left(1 \mathrm{H}, \mathrm{m}, \mathrm{C}^{5} \mathrm{H} H\right), 1.31-1.21(3 \mathrm{H}, \mathrm{m}$, $\left.\mathrm{C}^{6} \mathrm{H} H, \mathrm{C}^{7} \mathrm{H} H \& \mathrm{C}^{8} \mathrm{H} H\right) ;{ }^{13} \mathrm{C}$ NMR $\left(150 \mathrm{MHz}, \mathrm{MeOH}-\mathrm{d}_{4}\right) \delta 206.3$ $\left(C^{9} \mathrm{O}\right), 176.3(C \mathrm{OOH}), 173.7\left(C^{l} \mathrm{O}\right), 79.6\left(C^{3} \mathrm{HMe}\right), 49.6\left(C^{3 a} \mathrm{H}\right)$, ${ }_{50} 49.2\left(C^{8 a} \mathrm{H}\right), 49.0\left(C^{9 a} \mathrm{H}\right), 44.0\left(C^{4} \mathrm{H}\right), 41.5\left(C^{4 a} \mathrm{H}\right), 32.5\left(C^{5} \mathrm{H}_{2}\right)$, $26.7\left(C^{8} \mathrm{H}_{2}\right), 26.5\left(C^{6} \mathrm{H}_{2}\right), 26.1\left(C^{7} \mathrm{H}_{2}\right), 18.9\left(\mathrm{C}^{3} \mathrm{HCH}_{3}\right)$; IR (thin film) $2928(\mathrm{C}-\mathrm{H}), 2859(\mathrm{C}-\mathrm{H}), 1775(\mathrm{C}=\mathrm{O}), 1702(\mathrm{C}=\mathrm{O}), 1581$, 1416, 1201 (C-O), $1052 \mathrm{~cm}^{-1}$; m/z (EI) $267\left(100 \%,[\mathrm{M}+\mathrm{H}]^{+}\right)$; HRMS (EI) calcd for $\mathrm{C}_{14} \mathrm{H}_{18} \mathrm{O}_{5}[\mathrm{M}+\mathrm{H}]^{+}$266.1149, observed ${ }_{55} 266.1150$.
$(3 R, 3 \mathrm{a} R, 4 R, 4 \mathrm{a} R, 8 \mathrm{a} R, 9 \mathrm{a} S)-S$-Ethyl

3-methyl-1,9-

${ }_{60}$ dioxododecahydronaphtho[2,3-c]furan-4-carbothioate (5)

DCC (149 $\mathrm{mg}, 0.72 \mathrm{mmol}$ ) was added to a stirred solution of $(3 R, 3 \mathrm{a} S, 4 R, 4 \mathrm{a} R, 8 \mathrm{a} R, 9 \mathrm{a} S)-3$-methyl-1,9-

dioxododecahydronaphtho[2,3-c]furan-4-carboxylic acid (160 $\mathrm{mg}, 0.60 \mathrm{mmol})$, ethanethiol $(173 \mu \mathrm{L}, 2.40 \mathrm{mmol})$ and DMAP 65 (37.0 mg, $0.30 \mathrm{mmol})$ in $\mathrm{CH}_{2} \mathrm{Cl}_{2}(5 \mathrm{~mL})$ at $0{ }^{\circ} \mathrm{C}$, under Ar. The reaction mixture was stirred at $20{ }^{\circ} \mathrm{C}$ for $16 \mathrm{~h}$ before being filtered under vacuum to remove the precipitate. The filtrate was concentrated in vacuo to yield a crude colourless oil. Purification by flash column chromatography $\left(0-2 \% \mathrm{Et}_{2} \mathrm{O} / \mathrm{CH}_{2} \mathrm{Cl}_{2}\right)$ yielded $70(3 R, 3 \mathrm{a} R, 4 R, 4 \mathrm{a} R, 8 \mathrm{a} R, 9 \mathrm{a} S)-\mathrm{S}$-ethyl

3-methyl-1,9dioxododecahydronaphtho[2,3-c]furan-4-carbothioate (5) (128 $\mathrm{mg}, \quad 0.41 \mathrm{mmol}, 69 \%)$ as a white solid; Rf $0.64 \quad(8 \%$ $\left.\mathrm{Et}_{2} \mathrm{O} / \mathrm{CH}_{2} \mathrm{Cl}_{2}\right) ;{ }^{1} \mathrm{H} \mathrm{NMR}\left(600 \mathrm{MHz}, \mathrm{CDCl}_{3}\right) \delta 4.24(1 \mathrm{H}, \mathrm{dq}, J=$ $\left.10.5,6.0, \mathrm{C}^{3} H \mathrm{Me}\right), 3.73\left(1 \mathrm{H}, \mathrm{d}, J=8.7, \mathrm{C}^{9 \mathrm{a}} H\right), 2.96(1 \mathrm{H}, \mathrm{q}, J=$ $\left.756.5, \mathrm{SCH} \mathrm{HCH}_{3}\right), 2.92\left(1 \mathrm{H}, \mathrm{q}, J=6.5, \mathrm{SCH} H \mathrm{CH}_{3}\right), 2.84(1 \mathrm{H}, \mathrm{td}, J$ $\left.=11.9,3.4, \mathrm{C}^{8 \mathrm{a}} H\right), 2.75\left(1 \mathrm{H}, \mathrm{d}, J=4.5, \mathrm{C}^{4} H\right), 2.65(1 \mathrm{H}, \mathrm{dd}, J=$ $\left.10.5,8.7, \mathrm{C}^{3 \mathrm{a}} H\right), 2.00-1.95\left(1 \mathrm{H}, \mathrm{m}, \mathrm{C}^{8} \mathrm{HH}\right), 1.86-1.74(4 \mathrm{H}, \mathrm{m}$, $\left.\mathrm{C}^{4 \mathrm{a}} H, \mathrm{C}^{5} \mathrm{HH}, \mathrm{C}^{6} \mathrm{HH} \& \mathrm{C}^{7} H \mathrm{H}\right), 1.44\left(3 \mathrm{H}, \mathrm{d}, J=6.0, \mathrm{C}^{3} \mathrm{HCH}_{3}\right)$, $1.40-1.32\left(1 \mathrm{H}, \mathrm{m}, \mathrm{C}^{5} \mathrm{H} H\right), 1.30\left(3 \mathrm{H}, \mathrm{t}, J=7.3, \mathrm{SCH}_{2} \mathrm{CH}_{3}\right), 1.28$ ${ }_{80} 1.11\left(3 \mathrm{H}, \mathrm{m}, \mathrm{C}^{6} \mathrm{H} H, \mathrm{C}^{7} \mathrm{H} H \& \mathrm{C}^{8} \mathrm{H} H\right) ;{ }^{13} \mathrm{C}$ NMR $(150 \mathrm{MHz}$, $\left.\mathrm{CDCl}_{3}\right) \delta 203.8\left(C^{9} \mathrm{O}\right), 200.3$ (COSEt), $170.7 \quad\left(C^{l} \mathrm{O}\right), 77.3$ $\left(C^{3} \mathrm{HMe}\right), 54.7\left(C^{9 a} \mathrm{H}\right), 50.6\left(C^{4} \mathrm{H}\right), 48.8\left(C^{8 a} \mathrm{H}\right), 48.3\left(C^{3 a} \mathrm{H}\right)$, $41.8\left(C^{4 a} \mathrm{H}\right), 31.4\left(C^{5} \mathrm{H}_{2}\right), 25.7\left(C^{8} \mathrm{H}_{2}\right), 25.3\left(C^{6} \mathrm{H}_{2}\right), 24.8\left(C^{7} \mathrm{H}_{2}\right)$, $24.1\left(\mathrm{CH}_{2} \mathrm{CH}_{3}\right), 18.9\left(\mathrm{C}^{3} \mathrm{HCH}_{3}\right), 14.6\left(\mathrm{CH}_{2} \mathrm{CH}_{3}\right)$; IR (thin film) $852930(\mathrm{C}-\mathrm{H}), 2854(\mathrm{C}-\mathrm{H}), 1785(\mathrm{C}=\mathrm{O}), 1708(\mathrm{C}=\mathrm{O}), 1672,1197$ (C-O), 1059, $958 \mathrm{~cm}^{-1} ; \mathrm{m} / \mathrm{z}$ (EI) $252\left(100 \%,[\mathrm{M}-\mathrm{SEt}-\mathrm{Me}+\mathrm{OH}]^{+}\right)$; HRMS (EI) calcd for $\mathrm{C}_{16} \mathrm{H}_{22} \mathrm{O}_{4} \mathrm{~S}[\mathrm{M}+\mathrm{H}]^{+} 310.1233$, observed 310.1233 .

\section{$90(3 R, 3 a S, 4 a S, 8 a R, 9 a S)-3-M e t h y l-1,9-$} dioxododecahydronaphtho[2,3-c]furan-4-carbaldehyde (6) Triethylsilane $(263 \mu \mathrm{L}, 1.65 \mathrm{mmol})$ was added to a stirred suspension of $(3 R, 3 \mathrm{a} R, 4 R, 4 \mathrm{a} R, 8 \mathrm{a} R, 9 \mathrm{a} S)$-S-ethyl 3-methyl-1,9dioxododecahydronaphtho[2,3-c]furan-4-carbothioate (5) (128 $95 \mathrm{mg}, 0.41 \mathrm{mmol})$, palladium on carbon $(10 \%)(44.0 \mathrm{mg}, 0.04$ $\mathrm{mmol}$ ) and $\mathrm{MgSO}_{4}$ (to dry) in degassed acetone $(5 \mathrm{~mL})$ under Ar. The reaction mixture was stirred at $20{ }^{\circ} \mathrm{C}$ for $16 \mathrm{~h}$ before it was filtered over celite and concentrated in vacuo. Purification by flash column chromatography $\left(0-2 \% \mathrm{Et}_{2} \mathrm{O} / \mathrm{CH}_{2} \mathrm{Cl}_{2}\right)$ gave partial 100 racemisation at $\mathrm{C}^{4}$ to yield $(3 R, 3 \mathrm{a} S, 4 \mathrm{a} S, 8 \mathrm{a} R, 9 \mathrm{a} S)$-3-methyl-1,9dioxododecahydronaphtho[2,3-c]furan-4-carbaldehyde $(4 S: 4 R ; 1: 0.16)(50 \mathrm{mg}, 0.20 \mathrm{mmol}, 48 \%)$ as a colourless film; $\mathrm{Rf}$ $0.44\left(8 \% \mathrm{Et}_{2} \mathrm{O} / \mathrm{CH}_{2} \mathrm{Cl}_{2}\right) ;{ }^{1} \mathrm{H} \mathrm{NMR}\left(600 \mathrm{MHz}, \mathrm{CDCl}_{3}\right) \delta 10.14$ $(1 \mathrm{H}, \mathrm{s}, 4 S-\mathrm{CHO}), 4.29\left(1 \mathrm{H}, \mathrm{dq}, J=10.2,6.0,4 S-\mathrm{C}^{3} H \mathrm{Me}\right), 3.67$ $105\left(1 \mathrm{H}, \mathrm{d}, J=8.7,4 S-\mathrm{C}^{9 \mathrm{a}} H\right), 2.88\left(1 \mathrm{H}, \mathrm{dd}, J=10.2,8.7,4 S-\mathrm{C}^{3 \mathrm{a}} H\right)$, $2.80\left(1 \mathrm{H}, \mathrm{d}, J=4.9,4 S-\mathrm{C}^{4} H\right), 2.20(1 \mathrm{H}, \mathrm{ddd}, J=12.4,12.0,3.4$, $\left.4 S-\mathrm{C}^{8 \mathrm{a}} H\right), 2.09-1.96\left(3 \mathrm{H}, \mathrm{m}, 4 S-\mathrm{C}^{4 \mathrm{a}} H, 4 S-\mathrm{C}^{5} H \mathrm{H} \& 4 S-\mathrm{C}^{8} H \mathrm{H}\right)$, $1.88-1.81\left(2 \mathrm{H}, \mathrm{m}, 4 S-\mathrm{C}^{6} H \mathrm{H} \& 4 S-\mathrm{C}^{7} H \mathrm{H}\right), 1.68(1 \mathrm{H}, \mathrm{qd}, J=12.4$, $\left.3.4,4 S-\mathrm{C}^{5} \mathrm{H} H\right), 1.42\left(3 \mathrm{H}, \mathrm{d}, J=6.0, \mathrm{C}^{3} \mathrm{HCH}\right), 1.36-1.32(1 \mathrm{H}, \mathrm{m}$, $\left.1104 S-\mathrm{C}^{8} \mathrm{H} H\right), 1.23-1.17\left(2 \mathrm{H}, \mathrm{m}, 4 S-\mathrm{C}^{6} \mathrm{H} H \& 4 S-\mathrm{C}^{7} \mathrm{H} H\right) ;{ }^{13} \mathrm{C} \mathrm{NMR}$ $\left(150 \mathrm{MHz}, \mathrm{CDCl}_{3}\right) \delta 202.8\left(4 S-C^{9} \mathrm{O}\right), 202.7$ (4S-CHO), 170.5 $\left(4 S-C^{l} \mathrm{O}\right), 77.2\left(4 S-C^{3} \mathrm{HMe}\right), 54.5\left(4 S-C^{9 a} \mathrm{H}\right), 49.4\left(4 S-C^{8 a} \mathrm{H}\right)$, $49.1\left(4 S-C^{4} \mathrm{H}\right), 44.9\left(4 S-C^{3 a} \mathrm{H}\right), 41.6\left(4 S-C^{4 a} \mathrm{H}\right), 30.7\left(4 S-C^{5} \mathrm{H}_{2}\right)$, $26.0\left(4 S-C^{8} \mathrm{H}_{2}\right), 25.3 \quad\left(4 S-C^{6} \mathrm{H}_{2}\right), 24.8 \quad\left(4 S-C^{7} \mathrm{H}_{2}\right), 19.1 \quad(4 S-$ ${ }_{115} \mathrm{C}^{3} \mathrm{HCH}_{3}$ ). 
$(3 R, 3 \mathrm{a} S, 4 \mathrm{a} S, 8 \mathrm{a} R, 9 \mathrm{a} S)-3-M e t h y l-4-((E)-2-(5-(3-$ (trifluoromethyl)phenyl)pyridin-2-

yl)vinyl)octahydronaphtho[2,3-c]furan-1,9(3H,9aH)-dione $(8$ and 9)

$5 n$ - $\mathrm{BuLi}$ (1.6 $\mathrm{M}$ in hexanes) $(150 \mu \mathrm{L}, 0.24 \mathrm{mmol})$ was added dropwise to a stirred solution of diethyl (5-(3(trifluoromethyl)phenyl)pyridin-2-yl)methylphosphonate (7) (75 $\mathrm{mg}, 0.20 \mathrm{mmol})$ in anhydrous THF $(1 \mathrm{~mL})$ at $0{ }^{\circ} \mathrm{C}$, under Ar. The solution was stirred at $0 \quad{ }^{\circ} \mathrm{C}$ for $10 \mathrm{~min}$ before $10(3 R, 3 \mathrm{a} S, 4 \mathrm{a} S, 8 \mathrm{a} R, 9 \mathrm{a} S)-3$-methyl-1,9-

dioxododecahydronaphtho[2,3-c]furan-4-carbaldehyde (6) $\quad(50$ $\mathrm{mg}, 0.20 \mathrm{mmol}$ ) was added. The reaction mixture was stirred at 0 ${ }^{\circ} \mathrm{C}$ for $45 \mathrm{~min}$ and then quenched with sat. aq. $\mathrm{NH}_{4} \mathrm{Cl}(5 \mathrm{~mL})$. An extraction into EtOAc $(10 \mathrm{~mL})$ was done and the organic layer 15 was dried $\left(\mathrm{MgSO}_{4}\right)$, filtered and concentrated in vacuo to yield a crude yellow oil. Purification by flash column chromatography $\left(0-15 \% \mathrm{Et}_{2} \mathrm{O} / \mathrm{CH}_{2} \mathrm{Cl}_{2}\right)$ and then $\left(20-35 \% \mathrm{Et}_{2} \mathrm{O} /\right.$ toluene) yielded $(3 R, 3 \mathrm{a} S, 4 R, 4 \mathrm{a} S, 8 \mathrm{a} R, 9 \mathrm{a} S)-3-$ methyl-4-((E)-2-(5-(3-

(trifluoromethyl)phenyl)pyridin-2-

20 yl)vinyl)octahydronaphtho[2,3-c]furan-1,9(3H,9aH)-dione (8) (39 $\mathrm{mg}, 0.083 \mathrm{mmol}, 41 \%)$ and $(3 R, 3 \mathrm{a} S, 4 S, 4 \mathrm{a} S, 8 \mathrm{a} R, 9 \mathrm{a} S)-3-$ methyl-4-((E)-2-(5-(3-(trifluoromethyl)phenyl)pyridin-2-

yl)vinyl)octahydronaphtho[2,3-c]furan-1,9(3H,9aH)-dione

(14 mg, $0.03 \mathrm{mmol}, 15 \%)$ as white solids. Data for

$25(3 R, 3 \mathrm{a} S, 4 R, 4 \mathrm{a} S, 8 \mathrm{a} R, 9 \mathrm{a} S)-3-$ methyl-4-((E)-2-(5-(3-

(trifluoromethyl)phenyl)pyridin-2-

yl)vinyl)octahydronaphtho[2,3-c]furan-1,9(3H,9aH)-dione $\quad(8)$ : $[\alpha]_{\mathrm{D}}{ }^{20}=+14(\mathrm{MeOH}) ;{ }^{1} \mathrm{H}$ NMR $\left(600 \mathrm{MHz}, \mathrm{CDCl}_{3}\right) \delta 8.82(1 \mathrm{H}, \mathrm{d}$, $J=2.3, \mathrm{CHN}), 7.89(1 \mathrm{H}, \mathrm{dd}, J=8.3,2.3, \mathrm{CHCCHN}), 7.82(1 \mathrm{H}, \mathrm{s}$, $\left.{ }_{30} \mathrm{CHCCF}_{3}\right), 7.78\left(1 \mathrm{H}, \mathrm{d}, J=7.5, \mathrm{CHCCHCCF}_{3}\right), 7.68(1 \mathrm{H}, \mathrm{d}, J=$ 7.5, $\left.\mathrm{CHCHCCF}_{3}\right), 7.62\left(1 \mathrm{H}, \mathrm{t}, J=7.9, \mathrm{CHCHCCF}_{3}\right), 7.35(1 \mathrm{H}, \mathrm{d}$, $J=7.9, \mathrm{CHCN}), 7.10\left(1 \mathrm{H}, \mathrm{dd}, J=15.4,9.8, \mathrm{C}^{4} \mathrm{HCHCHPyr}\right), 6.66$ $\left(1 \mathrm{H}, \mathrm{d}, J=15.4, \mathrm{C}^{4} \mathrm{HCHCHPyr}\right), 4.42(1 \mathrm{H}, \mathrm{dq}, J=10.2,6$, $\left.\mathrm{C}^{3} H \mathrm{Me}\right), 3.66\left(1 \mathrm{H}, \mathrm{d}, J=8.7, \mathrm{C}^{9 \mathrm{a}} H\right), 2.71(1 \mathrm{H}, \mathrm{dd}, J=10.2,8.7$, $\left.{ }_{35} \mathrm{C}^{3 \mathrm{a}} H\right), 2.59\left(1 \mathrm{H}, \mathrm{dd}, J=9.6,4.0, \mathrm{C}^{4} H\right), 2.44(1 \mathrm{H}, \mathrm{td}, J=12.0$, 3.6, $\left.\mathrm{C}^{8 \mathrm{a}} H\right), 2.05-1.99\left(1 \mathrm{H}, \mathrm{m}, \mathrm{C}^{8} H \mathrm{H}\right), 1.88(1 \mathrm{H}, \mathrm{tt}, J=12.3,4$, $\left.\mathrm{C}^{4 \mathrm{a}} H\right), 1.82-1.77\left(1 \mathrm{H}, \mathrm{m}, \mathrm{C}^{7} H \mathrm{H}\right), 1.77-1.72\left(1 \mathrm{H}, \mathrm{m}, \mathrm{C}^{6} H \mathrm{H}\right), 1.71-$ $1.66\left(1 \mathrm{H}, \mathrm{m}, \mathrm{C}^{5} \mathrm{HH}\right), 1.48\left(3 \mathrm{H}, \mathrm{d}, J=6.0, \mathrm{C}^{3} \mathrm{HCH}_{3}\right), 1.46-1.40$ $\left(1 \mathrm{H}, \mathrm{m}, \mathrm{C}^{5} \mathrm{H} H\right), 1.40-1.34\left(1 \mathrm{H}, \mathrm{m}, \mathrm{C}^{8} \mathrm{H} H\right), 1.23-1.17(2 \mathrm{H}, \mathrm{m}$, $\left.{ }_{40} \mathrm{C}^{6} \mathrm{H} H \& \mathrm{C}^{7} \mathrm{H} H\right) ;{ }^{13} \mathrm{C}$ NMR $\left(150 \mathrm{MHz}, \mathrm{CDCl}_{3}\right) \delta 203.8\left(C^{9} \mathrm{O}\right)$, $170.9\left(C^{l} \mathrm{O}\right), 154.0(\mathrm{CN}), 148.3(\mathrm{CHN}), 138.4\left(\mathrm{CCHCCF}_{3}\right), 135.3$ (CHCCHN), 134.2 (CCHN), 132.8 ( $\left.\mathrm{C}^{4} \mathrm{HCHCHPyr}\right), 132.4$ $\begin{array}{lllll}\left(\mathrm{C}^{4} \mathrm{HCHCHPyr}\right), & 131.7 \quad\left(\mathrm{q}, \quad J=32.8, \quad C_{C F}\right), & 130.3\end{array}$ $\left(\mathrm{CHCCHCCF}_{3}\right), 129.8\left(\mathrm{CHCHCCF}_{3}\right), 125.0 \quad(\mathrm{q}, \quad J=4.0$, $\left.{ }_{45} \mathrm{CHCHCCF}_{3}\right), 123.8$ (q, $\left.J=4.0, \mathrm{CCHCCF}_{3}\right), 122.3$ (d, $J=247.6$, $\left.C \mathrm{~F}_{3}\right), 122.3(C \mathrm{HCN}), 78.0\left(C^{3} \mathrm{HMe}\right), 54.1\left(C^{9 a} \mathrm{H}\right), 51.8\left(C^{3 a} \mathrm{H}\right)$, $48.3\left(C^{8 a} \mathrm{H}\right), 42.2\left(C^{4 a} \mathrm{H}\right), 41.1\left(C^{4} \mathrm{H}\right), 31.8\left(C^{5} \mathrm{H}_{2}\right), 25.5\left(C^{6} \mathrm{H}_{2}\right)$, $25.4\left(C^{8} \mathrm{H}_{2}\right), 24.9\left(C^{7} \mathrm{H}_{2}\right), 19.2\left(\mathrm{C}^{3} \mathrm{HCH}_{3}\right)$; IR (thin film) $2921(\mathrm{C}-$ $\mathrm{H}), 2852(\mathrm{C}-\mathrm{H}), 1783(\mathrm{C}=\mathrm{O}), 1719(\mathrm{C}=\mathrm{O}), 1447,1335,1265$, 50 1167 (C-O), 1127, 804, $730 \mathrm{~cm}^{-1} ; \mathrm{m} / \mathrm{z}(\mathrm{ES}+) 470(100 \%$, $\left.[\mathrm{M}+\mathrm{H}]^{+}\right) ; \quad \mathrm{HRMS}(\mathrm{ES}+)$ calcd for $\mathrm{C}_{27} \mathrm{H}_{27} \mathrm{NO}_{3} \mathrm{~F}_{3} \quad[\mathrm{M}+\mathrm{H}]^{+}$ 470.1943, observed 470.1920. Data for $(3 R, 3 \mathrm{a} S, 4 S, 4 \mathrm{a} S, 8 \mathrm{a} R, 9 \mathrm{a} S)-$ 3-methyl-4-((E)-2-(5-(3-(trifluoromethyl)phenyl)pyridin-2-

yl)vinyl)octahydronaphtho[2,3-c]furan-1,9(3H,9aH)-dione $\quad(9)$ :

${ }_{55}{ }^{1} \mathrm{H}$ NMR $\left(600 \mathrm{MHz}, \mathrm{CDCl}_{3}\right) \delta 8.80(1 \mathrm{H}, \mathrm{d}, J=2.4, \mathrm{CHN}), 7.87$ $(1 \mathrm{H}, \mathrm{dd}, J=8.1,2.4, \mathrm{CHCCHN}), 7.81\left(1 \mathrm{H}, \mathrm{s}, \mathrm{CHCCF}_{3}\right), 7.76$ $\left(1 \mathrm{H}, \mathrm{d}, J=7.9, \mathrm{CHCCHCCF}_{3}\right), 7.67(1 \mathrm{H}, \mathrm{d}, J=7.9$, $\left.\mathrm{CHCHCCF}_{3}\right), 7.61\left(1 \mathrm{H}, \mathrm{t}, J=7.9, \mathrm{CHCHCCF}_{3}\right), 7.31(1 \mathrm{H}, \mathrm{d}, J=$
8.1, CHCN), $6.66\left(1 \mathrm{H}, \mathrm{d}, J=15.4, \mathrm{C}^{4} \mathrm{HCHCHPyr}\right), 6.60(1 \mathrm{H}, \mathrm{dd}$, $\left.{ }_{60} J=15.4,9.4, \mathrm{C}^{4} \mathrm{HCHCHPyr}\right), 4.57$ (1H, dq, $J=9.4,6.0, \mathrm{C}^{3} \mathrm{HMe}$ ), $3.58\left(1 \mathrm{H}, \mathrm{d}, J=7.9, \mathrm{C}^{9 \mathrm{a}} H\right), 2.90-2.83\left(2 \mathrm{H}, \mathrm{m}, \mathrm{C}^{3 \mathrm{a}} H \& \mathrm{C}^{4} H\right), 2.18$ $\left(1 \mathrm{H}, \mathrm{td}, J=11.6,3.2, \mathrm{C}^{8 \mathrm{a}} H\right), 1.99-1.92\left(2 \mathrm{H}, \mathrm{m}, \mathrm{C}^{5} H \mathrm{H} \& \mathrm{C}^{8} H \mathrm{H}\right)$, 1.86-1.81 (1H, m, $\left.\mathrm{C}^{6} H \mathrm{H}\right), 1.78-1.69\left(2 \mathrm{H}, \mathrm{m}, \mathrm{C}^{4 \mathrm{a}} H \& \mathrm{C}^{7} H \mathrm{H}\right), 1.44$ $\left(3 \mathrm{H}, \mathrm{d}, J=6.0, \mathrm{C}^{3} \mathrm{HCH}_{3}\right), 1.42-1.38\left(1 \mathrm{H}, \mathrm{m}, \mathrm{C}^{8} \mathrm{H} H\right), 1.24-1.16$ ${ }_{65}\left(2 \mathrm{H}, \mathrm{m}, \mathrm{C}^{6} \mathrm{H} H \& \mathrm{C}^{7} \mathrm{H} H\right), 1.11-1.01\left(1 \mathrm{H}, \mathrm{m}, \mathrm{C}^{5} \mathrm{H} H\right) ;{ }^{13} \mathrm{C} \mathrm{NMR}$ $\left(150 \mathrm{MHz}, \mathrm{CDCl}_{3}\right) \delta 203.9\left(C^{9} \mathrm{O}\right), 170.8\left(C^{l} \mathrm{O}\right), 153.9(C \mathrm{~N})$, $148.4(\mathrm{CHN}), 138.5\left(\mathrm{CCHCCF}_{3}\right), 135.2(\mathrm{CHCCHN}), 134.5$ (C $\left.{ }^{4} \mathrm{HCHCHPyr}\right), 134.1$ (CCHN), 131.9 (C $\left.\mathrm{C}^{4} \mathrm{HCHCHPyr}\right), 131.7$ $\left(\mathrm{q}, J=32.1, \mathrm{CCF}_{3}\right), 130.3\left(\mathrm{CHCCHCCF}_{3}\right), 129.8\left(\mathrm{CHCHCCF}_{3}\right)$, 70124.9 (q, $\left.J=3.8, \mathrm{CHCHCCF}_{3}\right), 123.8\left(\mathrm{q}, J=3.8, \mathrm{CCHCCF}_{3}\right)$, $124.1\left(\mathrm{~d}, J=271.7, C \mathrm{~F}_{3}\right), 122.0(C \mathrm{HCN}), 77.3\left(C^{3} \mathrm{HMe}\right), 56.7$ $\left(C^{9 a} \mathrm{H}\right), 53.1\left(C^{8 a} \mathrm{H}\right), 52.5\left(C^{3 a} \mathrm{H}\right), 45.5\left(C^{4} \mathrm{H}\right), 43.3\left(C^{4 a} \mathrm{H}\right), 33.1$ $\left(C^{5} \mathrm{H}_{2}\right), 25.3\left(C^{6} \mathrm{H}_{2}\right), 25.0\left(C^{8} \mathrm{H}_{2}\right), 24.8\left(C^{7} \mathrm{H}_{2}\right), 21.9\left(\mathrm{C}^{3} \mathrm{H} \mathrm{H}_{3}\right)$; IR (thin film) $2922(\mathrm{C}-\mathrm{H}), 2855(\mathrm{C}-\mathrm{H}), 1781(\mathrm{C}=\mathrm{O}), 1714(\mathrm{C}=\mathrm{O})$, $751440,1335,1268,1123$ (C-O), 1074, 810, $703 \mathrm{~cm}^{-1} ; \mathrm{m} / \mathrm{z}$ (EI) 469 $\left(100 \%,[\mathrm{M}]^{+}\right)$; HRMS (EI) calcd for $\mathrm{C}_{27} \mathrm{H}_{26} \mathrm{NO}_{3} \mathrm{~F}_{3}[\mathrm{M}]^{+} 469.1859$, observed 469.1859 .

$(3 R, 3 \mathrm{a} S, 4 R, 4 \mathrm{a} S, 8 \mathrm{a} R, 9 \mathrm{a} S)-9-H y d r o x y-3-m e t h y l-4-((E)-2-(5-(3-$ 80 (trifluoromethyl)phenyl)pyridin-2-

yl)vinyl)decahydronaphtho[2,3-c]furan-1(3H)-one (10 and 11)

$\mathrm{NaBH}_{4}(2.13 \mathrm{mg}, 0.06 \mathrm{mmol})$ was added to a stirred solution of $(3 R, 3 \mathrm{a} S, 4 R, 4 \mathrm{a} S, 8 \mathrm{a} R, 9 \mathrm{a} S)-3-$ methyl-4-((E)-2-(5-(3-

(trifluoromethyl)phenyl)pyridin-2-

85 yl)vinyl)octahydronaphtho[2,3-c]furan-1,9(3H,9aH)-dione $\quad$ (8) $(24.0 \mathrm{mg}, 0.05 \mathrm{mmol})$ in anhydrous THF $(2 \mathrm{~mL})$ and $\mathrm{MeOH}(2$ $\mathrm{mL})$ at $0{ }^{\circ} \mathrm{C}$, under Ar. The reaction mixture was stirred at $0{ }^{\circ} \mathrm{C}$ for $15 \mathrm{~min}$ and then quenched with sat. aq. $\mathrm{NH}_{4} \mathrm{Cl}(0.5 \mathrm{~mL})$. An extraction into $\mathrm{Et}_{2} \mathrm{O}(5 \mathrm{~mL})$ was done and the organic layer was 90 dried $\left(\mathrm{MgSO}_{4}\right)$, filtered and concentrated in vacuo to yield a crude yellow gum. Purification by flash column chromatography $\left(0-2 \% \quad \mathrm{Et}_{2} \mathrm{O} / \mathrm{CH}_{2} \mathrm{Cl}_{2}\right)$ yielded $(3 R, 3 \mathrm{a} S, 4 R, 4 \mathrm{a} S, 8 \mathrm{a} R, 9 R, 9 \mathrm{a} S)-9-$ hydroxy-3-methyl-4-((E)-2-(5-(3-

(trifluoromethyl)phenyl)pyridin-2-

$95 \mathrm{yl})$ vinyl)decahydronaphtho[2,3-c]furan-1(3H)-one as a mixture of isomers. Purification by preparative thin layer chomatography (0$\left.2 \% \quad \mathrm{Et}_{2} \mathrm{O} / \mathrm{CH}_{2} \mathrm{Cl}_{2}\right)$ yielded $(3 R, 3 \mathrm{a} S, 4 R, 4 \mathrm{a} S, 8 \mathrm{a} R, 9 R, 9 \mathrm{a} S)-9$ hydroxy-3-methyl-4-((E)-2-(5-(3-

(trifluoromethyl)phenyl)pyridin-2-

$100 \mathrm{yl})$ vinyl)decahydronaphtho[2,3-c]furan-1(3H)-one (10) $(9 \mathrm{mg}$, $0.02 \mathrm{mmol}, 38 \%)$ and $(3 R, 3 \mathrm{a} S, 4 R, 4 \mathrm{a} S, 8 \mathrm{a} R, 9 S, 9 \mathrm{a} S)-9$-hydroxy-3methyl-4-((E)-2-(5-(3-(trifluoromethyl)phenyl)pyridin-2-

yl)vinyl)decahydronaphtho[2,3-c]furan-1(3H)-one (11) (7 mg, 0.02 mmol, 29\%) as white solids. Data for 105 (3R,3aS,4R,4aS,8aR,9R,9aS)-9-hydroxy-3-methyl-4-((E)-2-(5-(3(trifluoromethyl)phenyl)pyridin-2-

yl)vinyl)decahydronaphtho[2,3-c]furan-1(3H)-one (10): ${ }^{1} \mathrm{H}$ NMR $\left(600 \mathrm{MHz}, \mathrm{CDCl}_{3}\right) \delta 8.80(1 \mathrm{H}, \mathrm{d}, J=1.9, \mathrm{CHN}), 7.86(1 \mathrm{H}, \mathrm{dd}, J$ $=8.1,1.9, \mathrm{CHCCHN}), 7.82\left(\mathrm{~s} 1 \mathrm{H}, \mathrm{CHCCF}_{3}\right), 7.77(1 \mathrm{H}, \mathrm{d}, J=$ $\left.1107.5, \mathrm{CHCCHCCF})_{3}\right), 7.66\left(1 \mathrm{H}, \mathrm{d}, J=7.5, \mathrm{CHCHCCF}_{3}\right), 7.61(1 \mathrm{H}$, $\left.\mathrm{t}, J=7.9, \mathrm{CHCHCCF}_{3}\right), 7.35(1 \mathrm{H}, \mathrm{d}, J=8.1, \mathrm{CHCN}), 6.88(1 \mathrm{H}$, $\left.\mathrm{dd}, J=15.4,9.8, \mathrm{C}^{4} \mathrm{HCHCHPyr}\right), 6.55(1 \mathrm{H}, \mathrm{d}, J=15.4$, $\left.\mathrm{C}^{4} \mathrm{HCHCHPyr}\right), 4.54\left(1 \mathrm{H}, \mathrm{dq}, J=10.4,6.0, \mathrm{C}^{3} \mathrm{HMe}\right), 3.47(1 \mathrm{H}$, ddd, $\left.\mathrm{J}=10.9,9.8,1.1, \mathrm{C}^{9} \mathrm{HOH}\right), 2.74\left(1 \mathrm{H}, \mathrm{dd}, J=9.8,7.9, \mathrm{C}^{9 \mathrm{a}} H\right)$, $1152.54(1 \mathrm{H}, \mathrm{d}, J=1.9, \mathrm{OH}), 2.40\left(1 \mathrm{H}, \mathrm{dd}, J=9.6,4.0, \mathrm{C}^{4} H\right), 2.36-$ $2.30\left(2 \mathrm{H}, \mathrm{m}, \mathrm{C}^{3 \mathrm{a}} H \& \mathrm{C}^{8} H \mathrm{H}\right), 1.78-1.72\left(2 \mathrm{H}, \mathrm{m}, \mathrm{C}^{6} H \mathrm{H} \& \mathrm{C}^{7} H \mathrm{H}\right)$, 
$1.58-1.49\left(3 \mathrm{H}, \mathrm{m}, \mathrm{C}^{4 \mathrm{a}} H, \mathrm{C}^{5} H \mathrm{H} \& \mathrm{C}^{8 \mathrm{a}} H\right), 1.47(1 \mathrm{H}, \mathrm{d}, J=6.0$, $\left.\mathrm{C}^{3} \mathrm{HCH}_{3}\right), 1.32-1.16\left(4 \mathrm{H}, \mathrm{m}, \mathrm{C}^{5} \mathrm{H} H, \mathrm{C}^{6} \mathrm{H} H, \mathrm{C}^{7} \mathrm{HH} \& \mathrm{C}^{8} \mathrm{H} H\right) ;{ }^{13} \mathrm{C}$ NMR $\left(150 \mathrm{MHz}, \mathrm{CDCl}_{3}\right) \delta 177.9\left(C^{l} \mathrm{O}\right), 154.6(C \mathrm{~N}), 148.1$ $(\mathrm{CHN}), \quad 131.8 \quad\left(\mathrm{CCHCCF}_{3}\right), \quad 131.5 \quad(\mathrm{CHCCHN}), \quad 131.3$ ${ }_{5}$ ( (C $\left.{ }^{4} \mathrm{HCHCHPyr}\right), \quad 130.9 \quad\left(\mathrm{q}, \quad J=32.1, \quad C \mathrm{CF}_{3}\right), \quad 130.3$

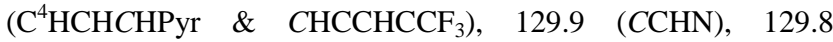
$\left(\mathrm{CHCHCCF}_{3}\right), 124.9$ (q, $\left.J=3.6, \mathrm{CHCHCCF}_{3}\right), 123.8$ (q, $J=3.6$, $\left.\mathrm{CCHCCF}_{3}\right), 124.1\left(\mathrm{~d}, J=272.1, C \mathrm{~F}_{3}\right), 121.6(\mathrm{CHCN}), 78.5$ $\left(C^{3} \mathrm{HMe}\right), 73.2\left(C^{9} \mathrm{HOH}\right), 49.5\left(C^{3 a} \mathrm{H}\right), 47.7\left(C^{9 a} \mathrm{H}\right), 41.1\left(C^{4} \mathrm{H}\right)$, $1040.2\left(C^{4 a} \mathrm{H}\right), 38.7\left(C^{8 a} \mathrm{H}\right), 32.1\left(C^{5} \mathrm{H}_{2}\right), 31.2\left(C^{8} \mathrm{H}_{2}\right), 26.1\left(C^{6} \mathrm{H}_{2}\right)$ $25.5\left(C^{7} \mathrm{H}_{2}\right), 19.5\left(\mathrm{C}^{3} \mathrm{HCH}_{3}\right)$; IR (thin film) $2921(\mathrm{C}-\mathrm{H}), 2851(\mathrm{C}-$ $\mathrm{H}), 1764(\mathrm{C}=\mathrm{O}), 1440,1334,1267,1166(\mathrm{C}-\mathrm{O}), 1124,1075$, $1051,804,755 \mathrm{~cm}^{-1} ; \mathrm{m} / \mathrm{z}(\mathrm{ES}+) 472\left(100 \%,[\mathrm{M}]^{+}\right)$; HRMS (EI) calcd for $\mathrm{C}_{27} \mathrm{H}_{28} \mathrm{NO}_{3} \mathrm{~F}_{3}[\mathrm{M}-\mathrm{H}]^{+}$471.2016, observed 471.2016. 15 Data for $(3 R, 3 \mathrm{a} S, 4 R, 4 \mathrm{a} S, 8 \mathrm{a} R, 9 S, 9 \mathrm{a} S)$-9-hydroxy-3-methyl-4-((E)2-(5-(3-(trifluoromethyl)phenyl)pyridin-2-

yl)vinyl)decahydronaphtho[2,3-c]furan-1(3H)-one (11): ${ }^{1} \mathrm{H}$ NMR $\left(600 \mathrm{MHz}, \mathrm{CDCl}_{3}\right) \delta 8.79(1 \mathrm{H}, \mathrm{d}, J=1.9, \mathrm{CHN}), 7.85(1 \mathrm{H}, \mathrm{dd}, J$ $=8.1,1.9, \mathrm{CHCCHN}), 7.81\left(1 \mathrm{H}, \mathrm{s}, \mathrm{CHCCF}_{3}\right), 7.76(1 \mathrm{H}, \mathrm{d}, J=$ 20 7.5, $\left.\mathrm{CHCHCCF}_{3}\right), 7.66\left(1 \mathrm{H}, \mathrm{d}, J=7.5, \mathrm{CHCCHCCF}_{3}\right), 7.61(1 \mathrm{H}$, $\left.\mathrm{t}, J=7.9, \mathrm{CHCHCCF}_{3}\right), 7.32(1 \mathrm{H}, \mathrm{d}, J=8.3, \mathrm{CHCN}), 6.87(1 \mathrm{H}$, $\left.\mathrm{dd}, J=15.4,10.2, \mathrm{C}^{4} \mathrm{HCHCHPyr}\right), 6.52(1 \mathrm{H}, \mathrm{d}, J=15.4$, $\left.\mathrm{C}^{4} \mathrm{HCHCHPyr}\right), 4.74\left(1 \mathrm{H}, \mathrm{dq}, J=10.5,6.0, \mathrm{C}^{3} \mathrm{HMe}\right), 4.09(1 \mathrm{H}$, td, $\left.J=4.9,2.3, \mathrm{C}^{9} H\right), 2.93\left(1 \mathrm{H}, \mathrm{dd}, J=8.3,4.9, \mathrm{C}^{9 \mathrm{a}} H\right), 2.48(1 \mathrm{H}$, $\left.{ }_{25} \mathrm{dd}, J=10.2,4.2, \mathrm{C}^{4} H\right), 2.21\left(1 \mathrm{H}, \mathrm{dd}, J=10.5,8.3, \mathrm{C}^{3 \mathrm{a}} H\right), 2.11$ $(1 \mathrm{H}, \mathrm{d}, J=4.9, \mathrm{OH}), 1.92\left(1 \mathrm{H}, \mathrm{tt}, J=11.7,4.5, \mathrm{C}^{4 \mathrm{a}} H\right), 1.78-1.70$ $\left(2 \mathrm{H}, \mathrm{m}, \mathrm{C}^{6} H \mathrm{H} \& \mathrm{C}^{7} H \mathrm{H}\right), 1.59-1.54\left(3 \mathrm{H}, \mathrm{m}, \mathrm{C}^{5} H \mathrm{H}, \mathrm{C}^{8} H \mathrm{H} \&\right.$ $\left.\mathrm{C}^{8 \mathrm{a}} H\right), 1.51-1.44\left(1 \mathrm{H}, \mathrm{m}, \mathrm{C}^{8} \mathrm{H} H\right), 1.44\left(3 \mathrm{H}, \mathrm{d}, J=6.0, \mathrm{C}^{3} \mathrm{HCH}_{3}\right)$, 1.31-1.19 (3H, m, $\left.\mathrm{C}^{5} \mathrm{H} H, \mathrm{C}^{6} \mathrm{HH} \& \mathrm{C}^{7} \mathrm{HH}\right) ;{ }^{13} \mathrm{C}$ NMR $(150 \mathrm{MHz}$, $\left.{ }_{30} \mathrm{CDCl}_{3}\right) \quad \delta \quad 177.4 \quad\left(C^{l} \mathrm{O}\right), \quad 154.7(\mathrm{CN}), 148.1(\mathrm{CHN}), 138.6$ $\left(\mathrm{CCHCCF}_{3}\right), 135.4\left(\mathrm{C}^{4} \mathrm{HCHCHPyr}\right), 135.1$ (CHCCHN), 133.7

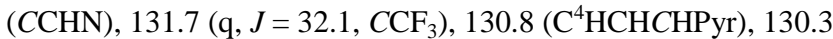
$\left(\mathrm{CHCCHCCF}_{3}\right), 129.8\left(\mathrm{CHCHCCF}_{3}\right), 124.8 \quad(\mathrm{q}, J=4.0$, $\left.\mathrm{CHCHCCF}_{3}\right), 123.8\left(\mathrm{q}, J=4.0, \mathrm{CCHCCF}_{3}\right), 124.1(\mathrm{~d}, J=271.7$, $\left.{ }_{35} C \mathrm{~F}_{3}\right), 121.9(C \mathrm{HCN}), 79.7\left(C^{3} \mathrm{HMe}\right), 69.0\left(C^{9} \mathrm{HOH}\right), 48.4\left(C^{3 a} \mathrm{H}\right)$, $45.8\left(C^{9 a} \mathrm{H}\right), 41.3\left(C^{4} \mathrm{H}\right), 38.4\left(C^{8 a} \mathrm{H}_{2}\right), 31.7\left(C^{4 a} \mathrm{H}\right), 31.4\left(C^{5} \mathrm{H}_{2}\right)$, $28.9\left(C^{8} \mathrm{H}_{2}\right), 26.4\left(C^{6} \mathrm{H}_{2}\right), 26.1\left(C^{7} \mathrm{H}_{2}\right), 19.3\left(\mathrm{C}^{3} \mathrm{HCH}_{3}\right)$; IR (thin film) $2924(\mathrm{C}-\mathrm{H}), 2853(\mathrm{C}-\mathrm{H}), 1761(\mathrm{C}=\mathrm{O}), 1440,1334,1267$, 1165 (C-O), 1124, 1075, 1050, 803, $700 \mathrm{~cm}^{-1} ; \mathrm{m} / \mathrm{z}(\mathrm{ES}+) 472$ $40\left(100 \%,[\mathrm{M}]^{+}\right)$; HRMS (ES+) calcd for $\mathrm{C}_{27} \mathrm{H}_{29} \mathrm{NO}_{3} \mathrm{~F}_{3}[\mathrm{M}]^{+}$ 472.2100, observed 472.2089.

\section{rac-tert-Butyl 1,9-dioxododecahydronaphtho[2,3-c]furan-4-} carboxylate (12 and 13)

$45 \mathrm{n}$-BuLi (2.7 $\mathrm{M}$ in heptanes) (12.8 $\mathrm{mL}, 34.5 \mathrm{mmol})$ was added dropwise to a stirred solution of freshly distilled TMP $(5.87 \mathrm{~mL}$, $34.5 \mathrm{mmol})$ in anhydrous 2-MeTHF $(40 \mathrm{~mL})$ at $-78^{\circ} \mathrm{C}$, under $\mathrm{N}_{2}$. The solution was warmed to $0{ }^{\circ} \mathrm{C}$ and then re-cooled to $-78{ }^{\circ} \mathrm{C}$. A solution of trans-methyl 2-(2-(tert-butoxy)-250 oxoethyl)cyclohexanecarboxylate (1) (4.21 g, $16.4 \mathrm{mmol})$ in anhydrous 2-MeTHF $(40 \mathrm{~mL})$ was added dropwise and stirring continued for $30 \mathrm{~min}$ at $-78{ }^{\circ} \mathrm{C}$. Next, furan-2(5H)-one $(0.58 \mathrm{~mL}$, $8.21 \mathrm{mmol})$ was added dropwise. The reaction mixture was stirred for a further $3 \mathrm{~h}$, quenched with sat. aq. $\mathrm{NH}_{4} \mathrm{Cl}(50 \mathrm{~mL})$ 55 and diluted with $\mathrm{H}_{2} \mathrm{O}(25 \mathrm{~mL})$. An extraction into EtOAc $(2 \times 50$ $\mathrm{mL}$ ) was done and the combined organic layers were washed with brine $(30 \mathrm{~mL})$, dried (hydrophobic frit) and concentrated in vacuo to yield a crude yellow oil with white precipitate. Purification by automated column chromatography (0-100\% 60 EtOAc/cyclohexane) yielded $\left(3 \mathrm{a} S^{*}, 4 R^{*}, 4 \mathrm{a} S^{*}, 8 \mathrm{a} S^{*}, 9 \mathrm{a} S^{*}\right)$-tertbutyl 1,9-dioxododecahydronaphtho[2,3-c]furan-4-carboxylate (13) (663 mg, $2.15 \mathrm{mmol}, 26 \%)$ and $\left(3 \mathrm{a} S^{*}, 4 R^{*}, 4 \mathrm{a} R^{*}, 8 \mathrm{a} R^{*}, 9 \mathrm{a} S^{*}\right)-$ tert-butyl 1,9-dioxododecahydronaphtho[2,3-c]furan-4carboxylate (12) (644 mg, $2.09 \mathrm{mmol}, 25 \%$ ) as white solids. Data 65 for $\quad\left(3 \mathrm{a} S^{*}, 4 R^{*}, 4 \mathrm{a} S^{*}, 8 \mathrm{a} S^{*}, 9 \mathrm{a} S^{*}\right)$-tert-butyl 1,9dioxododecahydronaphtho[2,3-c]furan-4-carboxylate (13): ${ }^{1} \mathrm{H}$ NMR $\left(600 \mathrm{MHz}, \mathrm{CDCl}_{3}\right) \delta{ }^{1} \mathrm{H}$ NMR $\left(600 \mathrm{MHz}, \mathrm{CDCl}_{3}\right) \delta 4.22$ $\left(1 \mathrm{H}, \mathrm{d}, J=3.8, \mathrm{C}^{3} \mathrm{H} H\right), 4.21\left(1 \mathrm{H}, \mathrm{s}, \mathrm{C}^{3} H \mathrm{H}\right), 3.49(1 \mathrm{H}, \mathrm{d}, J=7.5$, $\left.\mathrm{C}^{9 \mathrm{a}} H\right), 3.15\left(1 \mathrm{H}, \mathrm{ddd}, J=11.5,7.5,3.8, \mathrm{C}^{3 \mathrm{a}} H\right), 2.41(1 \mathrm{H}, \mathrm{t}, J=$ $\left.7011.5, \mathrm{C}^{4} H\right), 2.14-2.10\left(1 \mathrm{H}, \mathrm{m}, \mathrm{C}^{8} H \mathrm{H}\right), 1.99(1 \mathrm{H}, \mathrm{ddd}, J=12.0$, 10.7, 3.6, $\left.\mathrm{C}^{8 \mathrm{a}} H\right), 1.82-1.66\left(4 \mathrm{H}, \mathrm{m}, \mathrm{C}^{4 \mathrm{a}} H, \mathrm{C}^{5} H \mathrm{H}, \mathrm{C}^{6} H \mathrm{H} \&\right.$ $\left.\mathrm{C}^{7} \mathrm{HH}\right), 1.48\left(9 \mathrm{H}, \mathrm{s}, \mathrm{C}\left(\mathrm{CH}_{3}\right)_{3}\right), 1.46-1.41\left(1 \mathrm{H}, \mathrm{m}, \mathrm{C}^{8} \mathrm{H} H\right), 1.25-$ $1.12\left(3 \mathrm{H}, \mathrm{m}, \mathrm{C}^{5} \mathrm{H} H, \mathrm{C}^{6} \mathrm{H} H \& \mathrm{C}^{7} \mathrm{H} H\right) ;{ }^{13} \mathrm{C} \mathrm{NMR}(150 \mathrm{MHz}$, $\left.\mathrm{CDCl}_{3}\right) \delta 201.9\left(C^{9} \mathrm{O}\right), 172.4\left(C \mathrm{OO}^{\mathrm{t}} \mathrm{Bu}\right), 171.5\left(C^{l} \mathrm{O}\right), 82.5$ $75\left(C\left(\mathrm{CH}_{3}\right)_{3}\right), 70.5\left(C^{3} \mathrm{H}_{2}\right), 53.3\left(C^{9 a} \mathrm{H}\right), 51.3\left(C^{8 a} \mathrm{H}\right), 51.0\left(C^{4} \mathrm{H}\right)$, $42.8\left(C^{A a} \mathrm{H}\right), 42.8\left(C^{3 a} \mathrm{H}\right), 31.5\left(C^{8} \mathrm{H}_{2}\right), 28.2\left(\mathrm{C}\left(C \mathrm{CH}_{3}\right)_{3}\right), 25.1$ $\left(C^{5} \mathrm{H}_{2}\right), 25.0\left(C^{6} \mathrm{H}_{2}\right), 24.8\left(C^{7} \mathrm{H}_{2}\right)$; IR (thin film) $2974(\mathrm{C}-\mathrm{H})$, 2929 (C-H), $2856(\mathrm{C}-\mathrm{H}), 1795(\mathrm{C}=\mathrm{O}), 1715(\mathrm{C}=\mathrm{O}), 1367(\mathrm{C}-\mathrm{H})$, 1244 (C-O), 1157, 1021, $845 \mathrm{~cm}^{-1} ; \mathrm{m} / \mathrm{z}(\mathrm{ES}+) 326(100 \%$, $80 \mathrm{M}\left[\mathrm{NH}_{4}^{+}\right]^{+}$); HRMS (EI) calcd for $\mathrm{C}_{17} \mathrm{H}_{24} \mathrm{O}_{5}[\mathrm{M}+\mathrm{H}]^{+} 308.1618$, observed 308.1613. Data for $\left(3 \mathrm{a} S^{*}, 4 R^{*}, 4 \mathrm{a} R^{*}, 8 \mathrm{a} R^{*}, 9 \mathrm{a} S^{*}\right)$-tertbutyl 1,9-dioxododecahydronaphtho[2,3-c]furan-4-carboxylate (12): ${ }^{1} \mathrm{H}$ NMR $\left(600 \mathrm{MHz}, \mathrm{CDCl}_{3}\right) \delta 4.39\left(1 \mathrm{H}, \mathrm{t}, J=8.3, \mathrm{C}^{3} \mathrm{HH}\right)$, $3.94\left(1 \mathrm{H}, \mathrm{t}, J=9.8, \mathrm{C}^{3} \mathrm{H} H\right), 3.72\left(1 \mathrm{H}, \mathrm{d}, J=8.7, \mathrm{C}^{9 \mathrm{a}} H\right), 3.25(1 \mathrm{H}$, $\left.{ }_{85} \mathrm{ddd}, J=9.8,8.7,8.3, \mathrm{C}^{3 \mathrm{a}} H\right), 2.66\left(1 \mathrm{H}, \mathrm{td}, J=12.0,3.6, \mathrm{C}^{8 \mathrm{a}} H\right)$, $2.53\left(1 \mathrm{H}, \mathrm{d}, J=4.5, \mathrm{C}^{4} H\right), 2.03\left(1 \mathrm{H}, \mathrm{d}, J=14.7, \mathrm{C}^{8} H \mathrm{H}\right), 1.85$ $\left(1 \mathrm{H}\right.$, tdd, $\left.J=12.0,4.5,3.4, \mathrm{C}^{4 \mathrm{a}} H\right), 1.84-1.76\left(3 \mathrm{H}, \mathrm{m}, \mathrm{C}^{5} H \mathrm{H}\right.$, $\left.\mathrm{C}^{6} \mathrm{HH} \& \mathrm{C}^{7} \mathrm{HH}\right), 1.50\left(9 \mathrm{H}, \mathrm{s}, \mathrm{C}\left(\mathrm{CH}_{3}\right)_{3}\right), 1.49-1.42(1 \mathrm{H}, \mathrm{m}$, $\left.\mathrm{C}^{8} \mathrm{H} H\right), 1.36-1.15\left(3 \mathrm{H}, \mathrm{m}, \mathrm{C}^{5} \mathrm{H} H, \mathrm{C}^{6} \mathrm{H} H \& \mathrm{C}^{7} \mathrm{H} H\right) ;{ }^{13} \mathrm{C} \mathrm{NMR}$ $90\left(150 \mathrm{MHz}, \mathrm{CDCl}_{3}\right) \delta 203.8\left(C^{9} \mathrm{O}\right), 172.0\left(C \mathrm{OO}^{t} \mathrm{Bu}\right), 171.8\left(C^{l} \mathrm{O}\right)$, $82.5\left(C\left(\mathrm{CH}_{3}\right)_{3}\right), 69.5\left(C^{3} \mathrm{H}_{2}\right), 52.9\left(C^{9 a} \mathrm{H}\right), 48.2\left(C^{8 a} \mathrm{H}\right), 44.9$ $\left.\left(C^{4} \mathrm{H}\right), 40.6\left(C^{4 a} \mathrm{H}\right), 40.3\left(C^{3 a} \mathrm{H}\right), 31.4\left(C^{8} \mathrm{H}_{2}\right), 28.3\left(\mathrm{C}_{\left(C \mathrm{H}_{3}\right.}\right)_{3}\right)$, $25.9\left(C^{5} \mathrm{H}_{2}\right), 25.7\left(C^{6} \mathrm{H}_{2}\right), 25.1\left(C^{7} \mathrm{H}_{2}\right)$; IR (thin film) $2926(\mathrm{C}-\mathrm{H})$, $2850(\mathrm{C}-\mathrm{H}), 1778(\mathrm{C}=\mathrm{O}), 1715(\mathrm{C}=\mathrm{O}), 1698(\mathrm{C}=\mathrm{O}), 1365(\mathrm{C}-\mathrm{H})$, 951204 (C-O), 1138, 1002, $845 \mathrm{~cm}^{-1} ; \mathrm{m} / \mathrm{z}(\mathrm{ES}+) 326(100 \%$, $\left[\mathrm{M}+\mathrm{NH}_{4}\right]^{+}$); HRMS (ES-) calcd for $\mathrm{C}_{17} \mathrm{H}_{23} \mathrm{O}_{5}[\mathrm{M}-\mathrm{H}]^{+} 307.1545$, observed 307.1546.

\section{$\left(3 \mathrm{a} S^{*}, 4 R^{*}, 4 \mathrm{a} R^{*}, 8 \mathrm{a} R^{*}, 9 \mathrm{a} S^{*}\right)-1,9-$}

100 Dioxododecahydronaphtho[2,3-c]furan-4-carboxylic acid

TFA $(0.3 \mathrm{~mL})$ was added dropwise to a stirred solution of $\left(3 \mathrm{a} S^{*}, 4 R^{*}, 4 \mathrm{a} R^{*}, 8 \mathrm{a} R^{*}, 9 \mathrm{a} S^{*}\right)$-tert-butyl dioxododecahydronaphtho[2,3-c]furan-4-carboxylate (13) $(50.0$ $\mathrm{mg}, 0.16 \mathrm{mmol})$ in anhydrous $\mathrm{CH}_{2} \mathrm{Cl}_{2}(0.3 \mathrm{~mL})$ at $0{ }^{\circ} \mathrm{C}$, under $105 \mathrm{Ar}$. The reaction mixture was stirred at $20{ }^{\circ} \mathrm{C}$ for $16 \mathrm{~h}$ before being concentrated in vacuo. The residue was azeotroped with toluene to yield $\left(3 \mathrm{a} S^{*}, 4 R^{*}, 4 \mathrm{a} R^{*}, 8 \mathrm{a} R^{*}, 9 \mathrm{a} S^{*}\right)-1,9$ dioxododecahydronaphtho[2,3-c]furan-4-carboxylic acid (40.0 $\mathrm{mg}, 0.16 \mathrm{mmol}, 99 \%)$ as a white solid; ${ }^{1} \mathrm{H}$ NMR $(600 \mathrm{MHz}$, $\left.110 \mathrm{MeOH}-\mathrm{d}_{4}\right) \delta 4.43\left(1 \mathrm{H}, \mathrm{dd}, J=9.2,8.3, \mathrm{C}^{3} H \mathrm{H}\right), 4.05(1 \mathrm{H}, \mathrm{dd}, J=$ 9.8, 9.2, $\left.\mathrm{C}^{3} \mathrm{H} H\right), 3.75\left(1 \mathrm{H}, \mathrm{d}, J=9.0, \mathrm{C}^{9 \mathrm{a}} H\right), 3.37(1 \mathrm{H}, \mathrm{ddd}, J=$ 9.8, 9.0, 8.3, $\left.\mathrm{C}^{3 \mathrm{a}} H\right), 2.72\left(1 \mathrm{H}, \mathrm{d}, J=4.9, \mathrm{C}^{4} H\right), 2.69-2.64(1 \mathrm{H}, \mathrm{m}$, $\left.\mathrm{C}^{8 \mathrm{a}} H\right), 1.98-1.95\left(1 \mathrm{H}, \mathrm{m}, \mathrm{C}^{8} \mathrm{HH}\right), 1.98(1 \mathrm{H}$, dddd, $\mathrm{J}=13.2,12.0$, 4.9, 3.0, $\left.\mathrm{C}^{4 \mathrm{a}} H\right), 1.87-1.82\left(1 \mathrm{H}, \mathrm{m}, \mathrm{C}^{5} H \mathrm{H}\right), 1.82-1.75(2 \mathrm{H}, \mathrm{m}$, $\left.{ }_{115} \mathrm{C}^{6} \mathrm{HH} \& \mathrm{C}^{7} H \mathrm{H}\right), 1.44-1.35\left(1 \mathrm{H}, \mathrm{m}, \mathrm{C}^{5} \mathrm{H} H\right), 1.32-1.21(3 \mathrm{H}, \mathrm{m}$, $\left.\mathrm{C}^{6} \mathrm{H} H, \mathrm{C}^{7} \mathrm{H} H \& \mathrm{C}^{8} \mathrm{H} H\right) ;{ }^{13} \mathrm{C}$ NMR $\left(150 \mathrm{MHz}, \mathrm{MeOH}-\mathrm{d}_{4}\right) \delta 206.3$ 
$\left(C^{9} \mathrm{O}\right), 176.4(\mathrm{COOH}), 174.6\left(C^{l} \mathrm{O}\right), 71.3\left(C^{3} \mathrm{H}_{2}\right), 54.1\left(C^{9 a} \mathrm{H}\right)$, $49.4\left(C^{8 a} \mathrm{H}\right), 44.9\left(C^{4} \mathrm{H}\right), 41.2\left(C^{3 a} \mathrm{H}\right), 41.0\left(C^{4 a} \mathrm{H}\right), 32.5\left(C^{5} \mathrm{H}_{2}\right)$, $27.0\left(C^{8} \mathrm{H}_{2}\right), 26.7\left(C^{6} \mathrm{H}_{2}\right), 26.3\left(C^{7} \mathrm{H}_{2}\right)$; IR (thin film) $2936(\mathrm{C}-\mathrm{H})$, $2857(\mathrm{C}-\mathrm{H}), 1759(\mathrm{C}=\mathrm{O}), 1701(\mathrm{C}=\mathrm{O}), 1170,1000,839,800$, 5 719, 645, $549 \mathrm{~cm}^{-1} ; \mathrm{m} / \mathrm{z}(\mathrm{ES}+) 253\left(100 \%,[\mathrm{M}+\mathrm{H}]^{+}\right)$; HRMS (ES) calcd for $\mathrm{C}_{13} \mathrm{H}_{15} \mathrm{O}_{5}[\mathrm{M}-\mathrm{H}]^{+}$251.0920, observed 251.0919.

\section{$\left(3 \mathrm{a} R^{*}, 4 R^{*}, 4 \mathrm{a} R^{*}, 8 \mathrm{a} R^{*}, 9 \mathrm{a} S^{*}\right)-S$-ethyl}

\section{Dioxododecahydronaphtho[2,3-c]furan-4-carbothioate}

1,9-

DCC (29.7 $\mathrm{mg}, 0.14 \mathrm{mmol}$ ) was added to a stirred solution of $10\left(3 \mathrm{a} S^{*}, 4 R^{*}, 4 \mathrm{a} R^{*}, 8 \mathrm{a} R^{*}, 9 \mathrm{a} S^{*}\right)-1,9-$ dioxododecahydronaphtho[2,3-

c]furan-4-carboxylic acid (30.0 $\mathrm{mg}, 0.12 \mathrm{mmol})$, ethanethiol $(26.0 \mu \mathrm{L}, 0.36 \mathrm{mmol})$ and DMAP (7.00 $\mathrm{mg}, 0.06 \mathrm{mmol})$ in DMF $(1 \mathrm{~mL})$ at $0{ }^{\circ} \mathrm{C}$, under Ar. The reaction mixture was stirred at 20 ${ }^{\circ} \mathrm{C}$ for $2 \mathrm{~h}$ before being diluted with $\mathrm{CH}_{2} \mathrm{Cl}_{2}(10 \mathrm{~mL})$ and washed 15 with $\mathrm{H}_{2} \mathrm{O}(2 \times 5 \mathrm{~mL})$. The organic layer was dried $\left(\mathrm{MgSO}_{4}\right)$, filtered and concentrated in vacuo to yield a crude pink solid. Purification by column chromatography $\left(0-50 \% \mathrm{Et}_{2} \mathrm{O} / \mathrm{Pet}\right.$. Ether) yielded $\quad\left(3 \mathrm{a} R^{*}, 4 R^{*}, 4 \mathrm{a} R^{*}, 8 \mathrm{a} R^{*}, 9 \mathrm{a} S^{*}\right)-S$-ethyl 1,9 dioxododecahydronaphtho[2,3-c]furan-4-carbothioate $(10.5 \mathrm{mg}$, $200.035 \mathrm{mmol}, 29 \%)$ as a white solid; ${ }^{1} \mathrm{H} \mathrm{NMR}\left(600 \mathrm{MHz}, \mathrm{CDCl}_{3}\right)$ $\delta 4.38\left(1 \mathrm{H}, \mathrm{dd}, J=9.0,8.1, \mathrm{C}^{3} H \mathrm{H}\right), 3.93(1 \mathrm{H}, \mathrm{dd}, J=10.8,9.0$, $\left.\mathrm{C}^{3} \mathrm{H} H\right), 3.68\left(1 \mathrm{H}, \mathrm{d}, J=8.7, \mathrm{C}^{9 \mathrm{a}} H\right), 3.19(1 \mathrm{H}, \mathrm{ddd}, J=10.9,8.7$, $\left.8.1, \mathrm{C}^{3 \mathrm{a}} H\right), 2.94\left(2 \mathrm{H}, \mathrm{qd}, J=7.5,2.6, \mathrm{CH}_{2} \mathrm{CH}_{3}\right), 2.79(1 \mathrm{H}, \mathrm{td}, J=$ $\left.12.2,12.0,3.8, \mathrm{C}^{8 \mathrm{a}} H\right), 2.78\left(1 \mathrm{H}, \mathrm{d}, J=4.5, \mathrm{C}^{4} H\right), 2.02-1.97(1 \mathrm{H}$, $\left.25 \mathrm{~m}, \mathrm{C}^{8} H \mathrm{H}\right), 1.87\left(1 \mathrm{H}\right.$, dddd, $\left.J=12.2,12.0,4.5,3.8, \mathrm{C}^{4 \mathrm{a}} H\right), 1.83-$ $1.75\left(3 \mathrm{H}, \mathrm{m}, \mathrm{C}^{5} H \mathrm{H}, \mathrm{C}^{6} H \mathrm{H} \& \mathrm{C}^{7} H \mathrm{H}\right), 1.40-1.32\left(1 \mathrm{H}, \mathrm{m}, \mathrm{C}^{8} \mathrm{H} H\right)$, $1.30\left(3 \mathrm{H}, \mathrm{t}, J=7.5, \mathrm{CH}_{2} \mathrm{CH}_{3}\right), 1.28-1.21\left(1 \mathrm{H}, \mathrm{m}, \mathrm{C}^{5} \mathrm{H} H\right), 1.21-$ $1.15\left(2 \mathrm{H}, \mathrm{m}, \mathrm{C}^{6} \mathrm{H} H \& \mathrm{C}^{7} \mathrm{H} H\right) ;{ }^{13} \mathrm{C} \mathrm{NMR}\left(150 \mathrm{MHz}, \mathrm{CDCl}_{3}\right) \delta$ $203.7\left(C^{9} \mathrm{O}\right), 200.4$ (COSEt), $171.5\left(C^{l} \mathrm{O}\right), 69.1\left(C^{3} \mathrm{H}_{2}\right), 53.1$ ${ }_{30}\left(C^{9 a} \mathrm{H}\right), 51.4\left(C^{4} \mathrm{H}\right), 48.4\left(C^{8 a} \mathrm{H}\right), 41.2\left(C^{4 a} \mathrm{H}\right), 40.7\left(C^{3 a} \mathrm{H}\right), 31.5$ $\left(C^{5} \mathrm{H}_{2}\right), 25.7\left(C^{8} \mathrm{H}_{2}\right), 25.6\left(C^{6} \mathrm{H}_{2}\right), 24.8\left(C^{7} \mathrm{H}_{2}\right), 24.1\left(C_{2} \mathrm{CH}_{3}\right)$, $14.6\left(\mathrm{CH}_{2} \mathrm{CH}_{3}\right)$; IR (thin film) $2931(\mathrm{C}-\mathrm{H}), 2887(\mathrm{C}-\mathrm{H}), 1793$ $(\mathrm{C}=\mathrm{O}), 1708(\mathrm{C}=\mathrm{O}), 1672(\mathrm{C}=\mathrm{O}), 1203,1137,982,897 \mathrm{~cm}^{-1} ; \mathrm{m} / \mathrm{z}$ (ES+) $297\left(100 \%,[\mathrm{M}+\mathrm{H}]^{+}\right)$; HRMS $\left(\mathrm{ES}^{-}\right)$calcd for $\mathrm{C}_{15} \mathrm{H}_{19} \mathrm{O}_{4} \mathrm{~S}$ $35[\mathrm{M}-\mathrm{H}]^{+}$295.1003, observed 295.1004.

\section{rac-(3aS,4aS,8aR,9aS)-1,9-Dioxododecahydronaphtho[2,3- c]furan-4-carbaldehyde}

Triethylsilane $(15 \mu \mathrm{L}, 0.09 \mathrm{mmol})$ was added to a stirred 40 suspension of $\mathrm{rac}-(3 \mathrm{a} R, 4 R, 4 \mathrm{a} R, 8 \mathrm{a} R, 9 \mathrm{a} S)-S$-ethyl 1,9 dioxododecahydronaphtho[2,3-c]furan-4-carbothioate $(10.0 \mathrm{mg}$, $0.03 \mathrm{mmol})$, palladium on carbon $(10 \%)(4.00 \mathrm{mg}, 0.003 \mathrm{mmol})$ in degassed acetone $(0.2 \mathrm{~mL})$. The reaction mixture was stirred at $20{ }^{\circ} \mathrm{C}$ for $16 \mathrm{~h}$ before being filtered through celite and the filtrate 45 concentrated in vacuo. Purification by flash column chromatography $\left(0-2 \% \mathrm{Et}_{2} \mathrm{O} / \mathrm{CH}_{2} \mathrm{Cl}_{2}\right)$ gave partial racemisation at $\mathrm{C}^{4} \quad$ to yield $\quad \mathrm{rac}-(3 \mathrm{aS}, 4 \mathrm{aS}, 8 \mathrm{a} R, 9 \mathrm{aS})-1,9-$ dioxododecahydronaphtho[2,3-c]furan-4-carbaldehyde $\quad(4 S: 4 R$; 0.7:1) (7.00 mg, $0.03 \mathrm{mmol}, 99 \%)$ as a white solid; ${ }^{1} \mathrm{H}$ NMR $(600$ $\left.{ }_{50} \mathrm{MHz}, \mathrm{CDCl}_{3}\right) \delta 10.11(1 \mathrm{H}, \mathrm{s}, 4 S-\mathrm{CHO}), 9.90(1 \mathrm{H}, \mathrm{d}, J=1.5,4 R-$ $\mathrm{CHO}), 4.47-4.44\left(1 \mathrm{H}, \mathrm{m}, 4 R-\mathrm{C}^{3} H \mathrm{H}\right), 4.38(1 \mathrm{H}, \mathrm{dd}, J=9.0,7.9$, $\left.4 S-\mathrm{C}^{3} \mathrm{HH}\right), 4.04-3.96\left(2 \mathrm{H}, \mathrm{m}, 4 R-\mathrm{C}^{3} \mathrm{H} H \& 4 S-\mathrm{C}^{3} \mathrm{H} H\right), 3.61(1 \mathrm{H}$, $\left.\mathrm{d}, J=9.0,4 S-\mathrm{C}^{9 \mathrm{a}} H\right), 3.47-3.40\left(3 \mathrm{H}, \mathrm{m}, 4 R-\mathrm{C}^{9 \mathrm{a}} H, 4 R-\mathrm{C}^{3 \mathrm{a}} H \& 4 S-\right.$ $\left.\mathrm{C}^{3 \mathrm{a}} H\right), 3.09\left(1 \mathrm{H}, \mathrm{dd}, J=11.7,3.0,4 R-\mathrm{C}^{4} H\right), 2.83(1 \mathrm{H}, \mathrm{d}, J=4.5$, $\left.{ }_{55} 4 S-\mathrm{C}^{4} H\right), 2.22\left(1 \mathrm{H}, \mathrm{td}, J=11.7,3.4,4 R-\mathrm{C}^{8 \mathrm{a}} H\right), 2.17(1 \mathrm{H}, \mathrm{td}, J=$ $\left.11.7,3.8,4 S-C^{8 \mathrm{a}} H\right), 2.11-1.94\left(6 \mathrm{H}, \mathrm{m}, 4 R-\mathrm{C}^{4 \mathrm{a}} H, 4 S-\mathrm{C}^{4 \mathrm{a}} H, 4 R-\right.$
$\left.\mathrm{C}^{7} H \mathrm{H}, 4 S-\mathrm{C}^{7} H \mathrm{H}, 4 R-\mathrm{C}^{8} H \mathrm{H} \& 4 S-\mathrm{C}^{8} H \mathrm{H}\right), 1.90-1.74(3 \mathrm{H}, \mathrm{m}, 4 R-$ $\left.\mathrm{C}^{5} \mathrm{H} \mathrm{H}, 4 R-\mathrm{C}^{7} \mathrm{H} \mathrm{H} \& 4 S-\mathrm{C}^{7} H \mathrm{H}\right), 1.71-1.63\left(1 \mathrm{H}, \mathrm{m}, 4 S-\mathrm{C}^{5} H \mathrm{H}\right)$, 1.47-1.39 $\left(1 \mathrm{H}, \mathrm{m}, 4 R-\mathrm{C}^{8} \mathrm{H} H\right), 1.37-1.15\left(7 \mathrm{H}, \mathrm{m}, 4 R-\mathrm{C}^{5} \mathrm{H} H, 4 S-\right.$ $\left.{ }_{60} \mathrm{C}^{5} \mathrm{H} H, 4 R-\mathrm{C}^{6} \mathrm{H} H, 4 S-\mathrm{C}^{6} \mathrm{H} H, 4 R-\mathrm{C}^{7} \mathrm{H} H, 4 S-\mathrm{C}^{7} \mathrm{H} H \& 4 S-\mathrm{C}^{8} \mathrm{H} H\right)$; ${ }^{13} \mathrm{C}$ NMR $\left(150 \mathrm{MHz}, \mathrm{CDCl}_{3}\right) \delta 202.8\left(4 S-C^{9} \mathrm{O}\right), 202.8\left(4 R-C^{9} \mathrm{O}\right)$, 202.6 (4R-CHO), 201.3 (4S-CHO), $171.4\left(4 R-C^{l} \mathrm{O}\right), 171.0(4 S-$ $\left.C^{l} \mathrm{O}\right), 69.4\left(4 R-C^{3} \mathrm{H}_{2}\right), 68.0\left(4 S-C^{3} \mathrm{H}_{2}\right), 54.0\left(4 S-C^{9 a} \mathrm{H}\right), 52.9(4 S-$ $\left.C^{4} \mathrm{H}\right), 52.9\left(4 R-C^{9 a} \mathrm{H}\right), 52.6\left(4 S-C^{8 a} \mathrm{H}\right), 50.0\left(4 R-C^{4} \mathrm{H}\right), 49.4(4 R-$ $\left.{ }_{65} C^{8 a} \mathrm{H}\right), 41.0\left(4 R-C^{4 a} \mathrm{H}\right), 40.2\left(4 S-C^{4 a} \mathrm{H}\right), 39.0\left(4 S-C^{3 a} \mathrm{H}\right), 36.8(4 R-$ $\left.C^{3 a} \mathrm{H}\right), 32.0\left(4 S-C^{5} \mathrm{H}_{2}\right), 30.6\left(4 R-C^{5} \mathrm{H}_{2}\right), 26.0\left(4 R-C^{8} \mathrm{H}_{2}\right), 25.7(4 S-$ $\left.C^{8} \mathrm{H}_{2}\right), 24.9\left(4 S-C^{6} \mathrm{H}_{2} \& 4 R-C^{6} \mathrm{H}_{2}\right), 24.8\left(4 R-C^{7} \mathrm{H}_{2}\right), 24.6(4 S-$ $\left.C^{7} \mathrm{H}_{2}\right)$; IR (thin film) $2928(\mathrm{C}-\mathrm{H}), 2858(\mathrm{C}-\mathrm{H}), 1779(\mathrm{C}=\mathrm{O}), 1705$ $(\mathrm{C}=\mathrm{O}), 1208,1165,1120,1016,750 \mathrm{~cm}^{-1} ; \mathrm{m} / \mathrm{z}(\mathrm{ES}+) 237(100 \%$, $\left.70[\mathrm{M}+\mathrm{H}]^{+}\right)$; HRMS (EI) calcd for $\mathrm{C}_{13} \mathrm{H}_{16} \mathrm{O}_{4}[\mathrm{M}-\mathrm{H}]^{+}$236.1043, observed 236.1044.

rac-(3aS,4aS,8aR,9aS)-4-((E)-2-(5-(3(Trifluoromethyl)phenyl)pyridin-2-

75 yl)vinyl)octahydronaphtho[2,3-c]furan-1,9(3H,9aH)-dione (14 and 15)

$n$-BuLi (1.6 M in hexanes) (144 $\mu \mathrm{L}, 0.23 \mathrm{mmol})$ was added dropwise to a stirred solution of diethyl ((5-)(3(trifluoromethyl)phenyl)pyridin-2-yl)methyl)phosphonate (7) $80(79.0 \mathrm{mg}, 0.21 \mathrm{mmol})$ in anhydrous $\mathrm{THF}(1 \mathrm{~mL})$ at $0{ }^{\circ} \mathrm{C}$, under Ar. The solution was stirred at $0{ }^{\circ} \mathrm{C}$ for $10 \mathrm{~min}$ before a solution of $\quad r a c-(3 \mathrm{a} S, 4 \mathrm{a} S, 8 \mathrm{a} R, 9 \mathrm{a} S)$-1,9-dioxododecahydronaphtho[2,3c]furan-4-carbaldehyde (50 mg, $0.21 \mathrm{mmol}$ ) in anhydrous THF (1 $\mathrm{mL})$ was added. The reaction mixture was stirred at $0{ }^{\circ} \mathrm{C}$ for 45 $85 \mathrm{~min}$ and then quenched with sat. aq. $\mathrm{NH}_{4} \mathrm{Cl}(10 \mathrm{~mL})$. An extraction into EtOAc $(2 \times 5 \mathrm{~mL})$ was done and the combined organic layers were dried $\left(\mathrm{MgSO}_{4}\right)$, filtered and concentrated in vacuo to yield a crude yellow gum. Purification by flash column chromatography $\quad\left(0-2 \% \quad \mathrm{Et}_{2} \mathrm{O} / \mathrm{CH}_{2} \mathrm{Cl}_{2}\right)$ yielded rac90 $(3 \mathrm{a} S, 4 \mathrm{a} S, 8 \mathrm{a} R, 9 \mathrm{a} S)-4-((E)-2-(5-(3-$

(trifluoromethyl)phenyl)pyridin-2-

yl)vinyl)octahydronaphtho[2,3-c]furan-1,9(3H,9aH)-dione as a mixture of isomers. Purification by preparative TLC (0-2\% $\left.\mathrm{Et}_{2} \mathrm{O} / \mathrm{CH}_{2} \mathrm{Cl}_{2}\right)$ yielded $\left(3 \mathrm{a} S^{*}, 4 R^{*}, 4 \mathrm{a} S^{*}, 8 \mathrm{a} R^{*}, 9 \mathrm{a} S^{*}\right)-4-((E)-2-(5-(3-$ 95 (trifluoromethyl)phenyl)pyridin-2-

yl)vinyl)octahydronaphtho[2,3-c]furan-1,9(3H,9aH)-dione (14) (47.3 mg, $0.10 \mathrm{mmol}, 49 \%)$ and $\left(3 \mathrm{a} S^{*}, 4 S^{*}, 4 \mathrm{a} S^{*}, 8 \mathrm{a} R^{*}, 9 \mathrm{a} S^{*}\right)-4-$ ((E)-2-(5-(3-(trifluoromethyl)phenyl)pyridin-2-

yl)vinyl)octahydronaphtho[2,3-c]furan-1,9(3H,9aH)-dione (15) $100(24.5 \mathrm{mg}, \quad 0.05 \mathrm{mmol}, 26 \%)$ as white solids. Data for $\left(3 \mathrm{a} S^{*}, 4 R^{*}, 4 \mathrm{a} S^{*}, 8 \mathrm{a} R^{*}, 9 \mathrm{a} S^{*}\right)-4-((E)-2-(5-(3-$

(trifluoromethyl)phenyl)pyridin-2-

yl)vinyl)octahydronaphtho[2,3-c]furan-1,9(3H,9aH)-dione (14): ${ }^{1} \mathrm{H}$ NMR $\left(600 \mathrm{MHz}, \mathrm{CDCl}_{3}\right) \delta 8.82(1 \mathrm{H}, \mathrm{s}, \mathrm{CHN}), 7.88(1 \mathrm{H}, \mathrm{dd}, J$ $105=7.9,2.3, \mathrm{CHCCHN}), 7.82\left(1 \mathrm{H}, \mathrm{s}, \mathrm{CHCCF}_{3}\right), 7.77(1 \mathrm{H}, \mathrm{d}, J=$ 7.9, $\left.\mathrm{CHCCHCCF}_{3}\right), 7.67\left(1 \mathrm{H}, \mathrm{d}, J=7.5, \mathrm{CHCHCCF}_{3}\right), 7.62(1 \mathrm{H}$, $\left.\mathrm{t}, J=7.5, \mathrm{CHCHCCF}_{3}\right), 7.34(1 \mathrm{H}, \mathrm{d}, J=8.3, \mathrm{CHCN}), 7.08(1 \mathrm{H}$, $\left.\mathrm{dd}, J=15.4,9.8, \mathrm{C}^{4} \mathrm{HCHCHPyr}\right), 6.65(1 \mathrm{H}, \mathrm{d}, J=15.1$, $\left.\mathrm{C}^{4} \mathrm{HCHCHPyr}\right), 4.47\left(1 \mathrm{H}, \mathrm{t}, J=8.5, \mathrm{C}^{3} H \mathrm{H}\right), 4.11(1 \mathrm{H}, \mathrm{t}, J=9.8$, $\left.{ }_{10} \mathrm{C}^{3} \mathrm{H} H\right), 3.61\left(1 \mathrm{H}, \mathrm{d}, J=9.0, \mathrm{C}^{9 \mathrm{a}} H\right), 3.23(1 \mathrm{H}, \mathrm{ddd}, J=9.8,9.0$, $\left.8.5, \mathrm{C}^{3 \mathrm{a}} H\right), 2.60\left(1 \mathrm{H}, \mathrm{dd}, J=9.8,3.6, \mathrm{C}^{4} H\right), 2.42(1 \mathrm{H}, \mathrm{td}, J=$ 12.2, 3.6, $\left.\mathrm{C}^{8 \mathrm{a}} H\right), 2.07-2.02\left(1 \mathrm{H}, \mathrm{m}, \mathrm{C}^{8} H \mathrm{H}\right), 1.91(1 \mathrm{H}, \mathrm{tt}, J=12.2$, 3.6, $\left.\mathrm{C}^{4 \mathrm{a}} H\right), 1.83-1.78\left(1 \mathrm{H}, \mathrm{m}, \mathrm{C}^{7} H \mathrm{H}\right), 1.78-1.73\left(1 \mathrm{H}, \mathrm{m}, \mathrm{C}^{6} H \mathrm{H}\right)$, $1.72-1.67\left(1 \mathrm{H}, \mathrm{m}, \mathrm{C}^{5} \mathrm{HH}\right), 1.48-1.40\left(2 \mathrm{H}, \mathrm{m}, \mathrm{C}^{8} \mathrm{H} H\right), 1.40-1.34$ 
$\left(1 \mathrm{H}, \mathrm{m}, \mathrm{C}^{5} \mathrm{H} H\right), 1.23-1.15\left(2 \mathrm{H}, \mathrm{m}, \mathrm{C}^{6} \mathrm{H} H \& \mathrm{C}^{7} \mathrm{H} H\right) ;{ }^{13} \mathrm{C}$ NMR $\left(150 \mathrm{MHz}, \mathrm{CDCl}_{3}\right) \delta 203.8\left(C^{9} \mathrm{O}\right), 171.6\left(C^{l} \mathrm{O}\right), 154.0(\mathrm{CN})$, $148.2(\mathrm{CHN}), 138.4\left(\mathrm{CCHCCF}_{3}\right), 135.3(\mathrm{CHCCHN}), 134.2$ (CCHN), 132.7 ( $\left.\mathrm{C}^{4} \mathrm{HCHCHPyr}\right), 132.4\left(\mathrm{C}^{4} \mathrm{HCHCHPyr}\right), 131.8$ $5\left(\mathrm{q}, J=32.1, \mathrm{CCF}_{3}\right), 130.3\left(\mathrm{CHCCHCCF}_{3}\right), 129.8\left(\mathrm{CHCHCCF}_{3}\right)$, $125.0\left(\mathrm{q}, J=3.6, \mathrm{CCHCCF}_{3}\right), 123.8\left(\mathrm{q}, J=3.6, \mathrm{CHCHCCF}_{3}\right)$, $124.1\left(\mathrm{~d}, J=271.7, C \mathrm{~F}_{3}\right), 122.3(C \mathrm{HCN}), 70.0\left(C^{3} \mathrm{H}_{2}\right), 52.5$ $\left(C^{9 a} \mathrm{H}\right), 48.5\left(C^{8 a} \mathrm{H}\right), 43.5\left(C^{3 a} \mathrm{H}\right), 42.1\left(C^{4} \mathrm{H}\right), 41.6\left(C^{4 a} \mathrm{H}\right), 31.7$ $\left(C^{5} \mathrm{H}_{2}\right), 25.8\left(C^{8} \mathrm{H}_{2}\right), 25.5\left(C^{6} \mathrm{H}_{2}\right), 25.0\left(C^{7} \mathrm{H}_{2}\right)$; IR (thin film) $102930(\mathrm{C}-\mathrm{H}), 2855(\mathrm{C}-\mathrm{H}), 1784(\mathrm{C}=\mathrm{O}), 1708(\mathrm{C}=\mathrm{O}), 1478,1440$, 1335, 1164, 1124, 1018, $755 \mathrm{~cm}^{-1}$; m/z (ES+) $456(100 \%$, $[\mathrm{M}+\mathrm{H}]^{+}$); HRMS (ES) calcd for $\mathrm{C}_{26} \mathrm{H}_{23} \mathrm{NO}_{3} \mathrm{~F}_{3}[\mathrm{M}-\mathrm{H}]^{+}$454.1631, observed 454.1630. Chiral column purification (40\% EtOH/Heptane, $\mathrm{f}=30 \mathrm{ml} / \mathrm{min}$, Column $30 \mathrm{~mm} \times 25 \mathrm{~cm}$ Chiralcel $\left.{ }_{15} \mathrm{OJ}-\mathrm{H}\right) \quad$ gave $\quad(+)-(3 \mathrm{a} S, 4 R, 4 \mathrm{a} S, 8 \mathrm{a} R, 9 \mathrm{a} S)-4-((E)-2-(5-(3-$ (trifluoromethyl)phenyl)pyridin-2-

yl)vinyl)octahydronaphtho[2,3-c]furan-1,9(3H,9aH)-dione

$((+)-14)$ with an $[\alpha]_{\mathrm{D}}^{25}=+13.2 \quad(\mathrm{MeOH})$ and

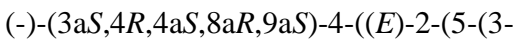

20 (trifluoromethyl)phenyl)pyridin-2-

yl)vinyl)octahydronaphtho[2,3-c]furan-1,9(3H,9aH)-dione

$((-)-14)$ with an $[\alpha]_{D}{ }^{25}=-13.4 \quad(\mathrm{MeOH})$. Data for $\left(3 \mathrm{a} S^{*}, 4 S^{*}, 4 \mathrm{a} S^{*}, 8 \mathrm{a} R^{*}, 9 \mathrm{a} S^{*}\right)-4-((E)-2-(5-(3-$

(trifluoromethyl)phenyl)pyridin-2-

$25 \mathrm{yl}$ )vinyl)octahydronaphtho[2,3-c]furan-1,9(3H,9aH)-dione (15):

${ }^{1} \mathrm{H}$ NMR $\left(600 \mathrm{MHz}, \mathrm{CDCl}_{3}\right) \delta 8.79(1 \mathrm{H}, \mathrm{d}, J=2.3, \mathrm{CHN}), 7.87$ $(1 \mathrm{H}, \mathrm{dd}, J=8.1,2.3, \mathrm{CHCCHN}), 7.81\left(1 \mathrm{H}, \mathrm{s}, \mathrm{CHCCF}_{3}\right), 7.76$ $\left(1 \mathrm{H}, \mathrm{d}, J=7.5, \mathrm{CHCCHCCF}_{3}\right), 7.67(1 \mathrm{H}, \mathrm{d}, J=7.5$, $\left.\mathrm{CHCHCCF}_{3}\right), 7.61\left(1 \mathrm{H}, \mathrm{t}, J=7.5, \mathrm{CHCHCCF}_{3}\right), 7.32(1 \mathrm{H}, \mathrm{d}, J=$ 30 8.3, CHCN), $6.70\left(1 \mathrm{H}, \mathrm{d}, J=15.4, \mathrm{C}^{4} \mathrm{HCHCHPyr}\right), 6.49(1 \mathrm{H}, \mathrm{dd}$, $\left.J=15.4,9.8, \mathrm{C}^{4} \mathrm{HCHCHPyr}\right), 4.43\left(1 \mathrm{H}, \mathrm{dd}, J=9.0,8.3, \mathrm{C}^{3} H \mathrm{H}\right)$, $4.15\left(1 \mathrm{H}, \mathrm{dd}, J=11.7,9.0, \mathrm{C}^{3} \mathrm{H} H\right), 3.51\left(1 \mathrm{H}, \mathrm{d}, J=8.7, \mathrm{C}^{9 \mathrm{a}} H\right)$, $3.32\left(1 \mathrm{H}\right.$, dddd, $\left.J=11.7,8.7,8.3,5.6, \mathrm{C}^{3 \mathrm{a}} H\right), 2.87(1 \mathrm{H}$, ddd, $J=$ $\left.11.3,9.8,5.6, \mathrm{C}^{4} H\right), 2.21\left(1 \mathrm{H}, \mathrm{td}, J=11.7,3.0, \mathrm{C}^{8 \mathrm{a}} H\right), 2.00-1.93$ ${ }_{35}\left(2 \mathrm{H}, \mathrm{m}, \mathrm{C}^{5} H \mathrm{H} \& \mathrm{C}^{8} H \mathrm{H}\right), 1.87-1.82\left(1 \mathrm{H}, \mathrm{m}, \mathrm{C}^{7} H \mathrm{H}\right), 1.75(2 \mathrm{H}, \mathrm{qd}$, $\left.J=11.3,3.4, \mathrm{C}^{4 a} H \& \mathrm{C}^{6} H \mathrm{H}\right), 1.49-1.40\left(1 \mathrm{H}, \mathrm{m}, \mathrm{C}^{8} \mathrm{H} H\right), 1.28-$ $1.15\left(2 \mathrm{H}, \mathrm{m}, \mathrm{C}^{6} \mathrm{H} H \& \mathrm{C}^{7} \mathrm{H} H\right), 1.13-1.05\left(1 \mathrm{H}, \mathrm{m}, \mathrm{C}^{5} \mathrm{H} H\right) ;{ }^{13} \mathrm{C}$ NMR $\left(150 \mathrm{MHz}, \mathrm{CDCl}_{3}\right) \delta 203.8\left(C^{9} \mathrm{O}\right), 171.7\left(C^{l} \mathrm{O}\right), 153.9$ $(\mathrm{CN}), 148.2(\mathrm{CHN}), 138.4\left(\mathrm{CCHCCF}_{3}\right), 135.2(\mathrm{CHCCHN}), 134.2$ $40(\mathrm{CCHN}), 133.2\left(\mathrm{C}^{4} \mathrm{HCHCHPyr}\right), 132.4\left(\mathrm{C}^{4} \mathrm{HCHCHPyr}\right), 131.8$ $\left(\mathrm{q}, J=32.1, \mathrm{CCF}_{3}\right), 130.3\left(\mathrm{CHCCHCCF}_{3}\right), 129.8\left(\mathrm{CHCHCCF}_{3}\right)$, 125.0 (q, $\left.J=3.6, \mathrm{CHCHCCF}_{3}\right), 123.8\left(\mathrm{q}, J=3.6, \mathrm{CCHCCF}_{3}\right)$, $124.7\left(\mathrm{~d}, J=272.0, C \mathrm{~F}_{3}\right), 122.0(\mathrm{CHCN}), 68.2\left(C^{3} \mathrm{H}_{2}\right), 54.7$ $\left(C^{9 a} \mathrm{H}\right), 52.9\left(C^{8 a} \mathrm{H}\right), 45.3\left(C^{4} \mathrm{H}\right), 44.6\left(C^{3 a} \mathrm{H}\right), 42.9\left(C^{4 a} \mathrm{H}\right), 33.0$ ${ }_{45}\left(C^{5} \mathrm{H}_{2}\right), 25.2\left(C^{8} \mathrm{H}_{2}\right), 24.9\left(C^{6} \mathrm{H}_{2}\right), 24.8\left(C^{7} \mathrm{H}_{2}\right)$; IR (thin film) $2923(\mathrm{C}-\mathrm{H}), 2853(\mathrm{C}-\mathrm{H}), 1782(\mathrm{C}=\mathrm{O}), 1703(\mathrm{C}=\mathrm{O}), 1333,1160$, 1119, 1074, 1010, 801, $699 \mathrm{~cm}^{-1} ; \mathrm{m} / \mathrm{z}(\mathrm{ES}+) 456(100 \%$, $[\mathrm{M}+\mathrm{H}]^{+}$); HRMS (ES) calcd for $\mathrm{C}_{26} \mathrm{H}_{23} \mathrm{NO}_{3} \mathrm{~F}_{3}[\mathrm{M}-\mathrm{H}]^{+}$454.1616, observed 454.1630. Chiral column purification (50\% 50 EtOH/Heptane, $\mathrm{f}=25 \mathrm{ml} / \mathrm{min}$, Column $22.1 \mathrm{~mm} \times 25 \mathrm{~cm}(\mathrm{R} ; \mathrm{R})$

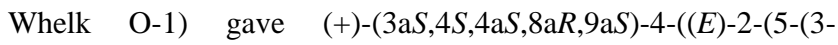
(trifluoromethyl)phenyl)pyridin-2-

yl)vinyl)octahydronaphtho[2,3-c]furan-1,9(3H,9aH)-dione

$((+)-15)$ with an $[\alpha]_{D}^{25}=+30.4 \quad(\mathrm{MeOH})$ and

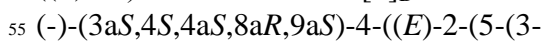

(trifluoromethyl)phenyl)pyridin-2-

yl)vinyl)octahydronaphtho[2,3-c]furan-1,9(3H,9aH)-dione $((-)-15)$ with an $[\alpha]_{\mathrm{D}}^{25}=-31.6(\mathrm{MeOH})$.
${ }_{60}\left(3 \mathrm{a} S^{*}, 4 R^{*}, 4 \mathrm{a} S^{*}, 8 \mathrm{a} S^{*}, 9 \mathrm{a} S^{*}\right)-1,9-$

\section{Dioxododecahydronaphtho[2,3-c]furan-4-carboxylic acid}

TFA (3.00 mL, $38.9 \mathrm{mmol})$ was added to a stirred solution of $\left(3 \mathrm{a} S^{*}, 4 R^{*}, 4 \mathrm{a} S^{*}, 8 \mathrm{a} S^{*}, 9 \mathrm{a} S^{*}\right)$-tert-butyl 1,9dioxododecahydronaphtho[2,3-c]furan-4-carboxylate (12) (1.09 $65 \mathrm{~g}, 3.53 \mathrm{mmol})$ in $\mathrm{CH}_{2} \mathrm{Cl}_{2}(6 \mathrm{~mL})$ at $0{ }^{\circ} \mathrm{C}$, under $\mathrm{N}_{2}$. The reaction mixture was stirred at $20^{\circ} \mathrm{C}$ for $16 \mathrm{~h}$ before being concentrated in vacuo. The residue was azeotroped with toluene $(25 \mathrm{~mL})$ to yield a crude pink solid. Purification by trituration $\left(\mathrm{CH}_{2} \mathrm{Cl}_{2}\right)$ yielded $\left(3 \mathrm{a} S^{*}, 4 R^{*}, 4 \mathrm{a} S^{*}, 8 \mathrm{a} S^{*}, 9 \mathrm{a} S^{*}\right)$-1,9-dioxododecahydronaphtho[2,3-

70 c]furan-4-carboxylic acid (441 mg, $1.75 \mathrm{mmol}, 49 \%$ ) as a white solid; $1 \mathrm{H} \mathrm{NMR}\left(600 \mathrm{MHz}, \mathrm{CDCl}_{3}\right) \delta 4.33\left(1 \mathrm{H}, \mathrm{d}, J=9.8, \mathrm{C}^{3} \mathrm{HH}\right)$, $4.27\left(1 \mathrm{H}, \mathrm{dd}, J=9.8,4.5, \mathrm{C}^{3} \mathrm{H} H\right), 3.55\left(1 \mathrm{H}, \mathrm{d}, J=7.6, \mathrm{C}^{9 \mathrm{a}} H\right)$, $3.22\left(1 \mathrm{H}\right.$, ddd, $\left.J=11.5,7.6,4.5, \mathrm{C}^{3 \mathrm{a}} H\right), 2.57(1 \mathrm{H}, \mathrm{t}, J=11.5$, $\left.\mathrm{C}^{4} H\right), 2.19-2.12\left(1 \mathrm{H}, \mathrm{m}, \mathrm{C}^{8} H \mathrm{H}\right), 2.06-2.01\left(1 \mathrm{H}, \mathrm{m}, \mathrm{C}^{8 \mathrm{a}} H\right), 1.89-$ $751.82\left(2 \mathrm{H}, \mathrm{m}, \mathrm{C}^{6} H \mathrm{H} \& \mathrm{C}^{7} H \mathrm{H}\right), 1.81-1.73\left(2 \mathrm{H}, \mathrm{m}, \mathrm{C}^{5} H \mathrm{H} \& \mathrm{C}^{4 \mathrm{a}} H\right)$, 1.26-1.18 (4H, m, $\left.\mathrm{C}^{5} \mathrm{H} H, \mathrm{C}^{6} \mathrm{H} H, \mathrm{C}^{7} \mathrm{H} H \& \mathrm{C}^{8} \mathrm{H} H\right) ;{ }^{13} \mathrm{C}$ NMR $(150$ $\left.\mathrm{MHz}, \mathrm{CDCl}_{3}\right) \delta 201.5\left(C^{9} \mathrm{O}\right), 177.5(C \mathrm{OOH}), 171.2\left(C^{l} \mathrm{O}\right), 70.6$ $\left(C^{3} \mathrm{H}_{2}\right), 53.2\left(C^{9 a} \mathrm{H}\right), 51.1\left(C^{4} \mathrm{H}\right), 49.9\left(C^{8 a} \mathrm{H}\right), 42.5\left(C^{3 a} \mathrm{H}\right), 42.4$ $\left(C^{A a} \mathrm{H}\right), 31.8\left(C^{5} \mathrm{H}_{2}\right), 25.1\left(C^{8} \mathrm{H}_{2}\right), 25.0\left(C^{6} \mathrm{H}_{2}\right), 24.7\left(C^{7} \mathrm{H}_{2}\right)$; IR 80 (thin film) $2930(\mathrm{C}-\mathrm{H}), 2869(\mathrm{C}-\mathrm{H}), 1779(\mathrm{C}=\mathrm{O}), 1705(\mathrm{C}=\mathrm{O})$, 1171, 1166, $1018 \mathrm{~cm}^{-1} ; \mathrm{m} / \mathrm{z}(\mathrm{ES}+) 253\left(100 \%,[\mathrm{M}+\mathrm{H}]^{+}\right) ; \mathrm{HRMS}$ (EI) calcd for $\mathrm{C}_{13} \mathrm{H}_{16} \mathrm{O}_{5}[\mathrm{M}]^{+}$252.0992, observed 252.0982.

\section{$\left(3 \mathrm{a} R^{*}, 4 R^{*}, 4 \mathrm{a} S^{*}, 8 \mathrm{a} S^{*}, 9 \mathrm{a} S^{*}\right)-S$-ethyl}

1,9-

\section{${ }_{85}$ Dioxododecahydronaphtho[2,3-c]furan-4-carbothioate}

$\mathrm{EDCl}(502 \mathrm{mg}, 2.62 \mathrm{mmol})$ was added to a stirred solution of (3a $\left.S^{*}, 4 R^{*}, 4 \mathrm{a} S^{*}, 8 \mathrm{a} S^{*}, 9 \mathrm{a} S^{*}\right)-1,9-$ dioxododecahydronaphtho[2,3c]furan-4-carboxylic acid (440 mg, $1.74 \mathrm{mmol})$, ethanethiol $(0.52$ $\mathrm{mL}, 6.98 \mathrm{mmol})$ and DMAP (21.3 $\mathrm{mg}, 0.17 \mathrm{mmol})$ in $\mathrm{CH}_{2} \mathrm{Cl}_{2}(20$ $90 \mathrm{~mL}$ ) under $\mathrm{N}_{2}$. The reaction mixture was stirred at $20{ }^{\circ} \mathrm{C}$ for $2 \mathrm{~h}$ and then quenched with sat. aq. $\mathrm{NH}_{4} \mathrm{Cl}(10 \mathrm{~mL})$. An extraction into $\mathrm{CH}_{2} \mathrm{Cl}_{2}(10 \mathrm{~mL})$ was done and the organic layer was dried (hydrophobic frit) and concentrated in vacuo to yield $\left(3 \mathrm{a} R^{*}, 4 R^{*}, 4 \mathrm{a} S^{*}, 8 \mathrm{a} S^{*}, 9 \mathrm{a} S^{*}\right)-S$-ethyl

$1,9-$

95 dioxododecahydronaphtho[2,3-c]furan-4-carbothioate $(482 \mathrm{mg}$, $1.62 \mathrm{mmol}, 93 \%)$ as an off-white solid; ${ }^{1} \mathrm{H}$ NMR $(600 \mathrm{MHz}$, $\left.\mathrm{CDCl}_{3}\right) \delta 4.38\left(1 \mathrm{H}, \mathrm{d}, J=9.6, \mathrm{C}^{3} \mathrm{HH}\right), 4.18(1 \mathrm{H}, \mathrm{dd}, J=9.6,4.7$, $\left.\mathrm{C}^{3} \mathrm{H} H\right), 3.51\left(1 \mathrm{H}, \mathrm{d}, J=7.5, \mathrm{C}^{9 \mathrm{a}} H\right), 3.21(1 \mathrm{H}, \mathrm{ddd}, J=11.6,7.4$, 4.7, $\left.\mathrm{C}^{3 \mathrm{a}} \mathrm{H}\right), 3.02-2.89\left(2 \mathrm{H}, \mathrm{m}, \mathrm{CH}_{2} \mathrm{CH}_{3}\right), 2.70(1 \mathrm{H}, \mathrm{t}, J=11.1$, $\left.{ }_{100} \mathrm{C}^{4} H\right), 2.15-2.10\left(1 \mathrm{H}, \mathrm{m}, \mathrm{C}^{8} H \mathrm{H}\right), 2.02(1 \mathrm{H}, \mathrm{td}, J=11.2,3.2$, $\left.\mathrm{C}^{8 \mathrm{a}} H\right), 1.85-1.69\left(4 \mathrm{H}, \mathrm{m}, \mathrm{C}^{4 \mathrm{a}} H, \mathrm{C}^{5} H \mathrm{H}, \mathrm{C}^{6} H \mathrm{H} \& \mathrm{C}^{7} H \mathrm{H}\right), 1.30-$ 1.27 (3H, m, $\left.\mathrm{CH}_{2} \mathrm{CH}_{3}\right), 1.27-1.09\left(4 \mathrm{H}, \mathrm{m}, \mathrm{C}^{5} \mathrm{H} H, \mathrm{C}^{6} \mathrm{H} H, \mathrm{C}^{7} \mathrm{H} H\right.$ \& $\left.\mathrm{C}^{8} \mathrm{H} H\right)$; IR (thin film) $2930(\mathrm{C}-\mathrm{H}), 2876(\mathrm{C}-\mathrm{H}), 1783(\mathrm{C}=\mathrm{O})$, $1706(\mathrm{C}=\mathrm{O}), 1671(\mathrm{C}=\mathrm{O}), 1448,1362,1203,1137,1012,981$, $105896 \mathrm{~cm}^{-1} ; \mathrm{m} / \mathrm{z}(\mathrm{ES}+) 297\left(100 \%,[\mathrm{M}+\mathrm{H}]^{+}\right)$; HRMS (EI) calcd for $\mathrm{C}_{15} \mathrm{H}_{20} \mathrm{O}_{4} \mathrm{~S}[\mathrm{M}]^{+}$296.1077, observed 296.1076.

\section{rac-(3aS,4aR,8aS,9aS)-1,9-Dioxododecahydronaphtho[2,3- c]furan-4-carbaldehyde}

110 Triethylsilane $(1.02 \mathrm{~mL}, 6.41 \mathrm{mmol})$ was added to a stirred solution of $\left(3 \mathrm{a} R^{*}, 4 R^{*}, 4 \mathrm{a} S^{*}, 8 \mathrm{a} S^{*}, 9 \mathrm{a} S^{*}\right)-S$-ethyl 1,9dioxododecahydronaphtho[2,3-c]furan-4-carbothioate $(475 \mathrm{mg}$, $1.60 \mathrm{mmol})$, palladium on carbon $(10 \%)(171 \mathrm{mg}, 1.60 \mathrm{mmol})$ and $\mathrm{MgSO}_{4}$ (to dry) in anhydrous, degassed acetone $(25 \mathrm{~mL})$ 115 under $\mathrm{N}_{2}$. The reaction mixture was stirred at $20{ }^{\circ} \mathrm{C}$ for $16 \mathrm{~h}$ before being filtered through celite and the filtrate concentrated in 
vacuo. Purification by flash column chromatography (0-100\% EtOAc/cyclohexane) gave partial racemisation at $\mathrm{C}^{4}$ to yield $\mathrm{rac}$ (3aS,4aR,8aS,9aS)-1,9-dioxododecahydronaphtho[2,3-c]furan-4carbaldehyde $(4 S: 4 R ; 1: 6)$ (130 $\mathrm{mg}, 0.55 \mathrm{mmol}, 34 \%)$ as a 5 colourless oil; ${ }^{1} \mathrm{H}$ NMR $\left(600 \mathrm{MHz}, \mathrm{CDCl}_{3}\right) \delta 9.90-9.89(1 \mathrm{H}, \mathrm{m}$, $4 S$-CHO), $9.83(1 \mathrm{H}, \mathrm{d}, J=2.8,4 R-\mathrm{CHO}), 4.26(1 \mathrm{H}, \mathrm{dd}, J=9.8$, $\left.4.7,4 R-\mathrm{C}^{3} \mathrm{HH}\right), 4.20\left(1 \mathrm{H}, \mathrm{d}, J=9.6,4 R-\mathrm{C}^{3} \mathrm{H} H\right), 3.56(1 \mathrm{H}, \mathrm{d}, J=$ $\left.7.8,4 R-\mathrm{C}^{9 \mathrm{a}} H\right), 3.22\left(1 \mathrm{H}\right.$, ddd, $\left.J=11.6,7.5,4.5,4 R-\mathrm{C}^{3 \mathrm{a}} H\right), 2.57$ $\left(1 \mathrm{H}, \mathrm{td}, J=11.4,2.9,4 R-\mathrm{C}^{4} H\right), 2.18-2.11\left(2 \mathrm{H}, \mathrm{m}, 4 R-\mathrm{C}^{8} H \mathrm{H} \&\right.$ $\left.{ }_{10} 4 R-\mathrm{C}^{8 \mathrm{a}} H\right), 2.04-1.97\left(2 \mathrm{H}, \mathrm{m}, 4 R-\mathrm{C}^{6} H \mathrm{H} \& 4 R-\mathrm{C}^{7} H \mathrm{H}\right), 1.89-1.77$ $\left(2 \mathrm{H}, \mathrm{m}, 4 R-\mathrm{C}^{5} H \mathrm{H} \& 4 R-\mathrm{C}^{4 \mathrm{a}} H\right), 1.40-1.35\left(1 \mathrm{H}, \mathrm{m}, 4 R-\mathrm{C}^{8} \mathrm{H} H\right)$, $1.32-1.19\left(3 \mathrm{H}, \mathrm{m}, 4 R-\mathrm{C}^{5} \mathrm{H} H, 4 R-\mathrm{C}^{6} \mathrm{H} H \& 4 R-\mathrm{C}^{7} \mathrm{H} H\right) ;{ }^{13} \mathrm{C} \mathrm{NMR}$ $\left(150 \mathrm{MHz}, \mathrm{CDCl}_{3}\right) \delta 201.6\left(4 R-C^{9} \mathrm{O}\right), 201.3(4 R-C \mathrm{HO}), 168.7$ $\left(4 R-C^{l} \mathrm{O}\right), 70.4\left(4 R-C^{3} \mathrm{H}_{2}\right), 54.8\left(4 R-C^{4} \mathrm{H}\right), 52.9\left(4 R-C^{9 a} \mathrm{H}\right), 51.1$ $15\left(4 R-C^{8 a} \mathrm{H}\right), 41.8\left(4 R-C^{4 a} \mathrm{H}\right), 39.1\left(4 R-C^{3 a} \mathrm{H}\right), 31.7\left(4 R-C^{5} \mathrm{H}_{2}\right), 24.9$ $\left(4 R-C^{8} \mathrm{H}_{2} \& 4 \mathrm{R}-C^{6} \mathrm{H}_{2}\right), 24.5\left(4 R-C^{7} \mathrm{H}_{2}\right), 19.1\left(4 S-\mathrm{C}^{3} \mathrm{HCH}_{3}\right)$; IR (thin film) $2927(\mathrm{C}-\mathrm{H}), 2855(\mathrm{C}-\mathrm{H}), 1770(\mathrm{C}=\mathrm{O}), 1713(\mathrm{C}=\mathrm{O})$, 1125, $730 \mathrm{~cm}^{-1} ; \mathrm{m} / \mathrm{z}(\mathrm{ES}+) 237\left(100 \%,[\mathrm{M}+\mathrm{H}]^{+}\right)$; HRMS (EI) calcd for $\mathrm{C}_{13} \mathrm{H}_{16} \mathrm{O}_{4}[\mathrm{M}]^{+}$236.1043, observed 236.1049.

20

$\left(3 \mathrm{a} S^{*}, 4 R^{*}, 4 \mathrm{a} R *, 8 \mathrm{a} S^{*}, 9 \mathrm{a} S^{*}\right)-4-((E)-2-(5-(3-$ (Trifluoromethyl)phenyl)pyridin-2yl)vinyl)octahydronaphtho[2,3-c]furan-1,9(3H,9aH)-dione (16)

$25 n$-BuLi (2.7 $\mathrm{M}$ in heptanes) $(0.25 \mathrm{~mL}, 0.66 \mathrm{mmol})$ was added dropwise to a stirred solution of diethyl (5-(3(trifluoromethyl)phenyl)pyridin-2-yl)methylphosphonate (7) (226 $\mathrm{mg}, 0.60 \mathrm{mmol})$ in anhydrous 2-MeTHF $(10 \mathrm{~mL})$ at $0{ }^{\circ} \mathrm{C}$, under $\mathrm{N}_{2}$. The solution was stirred at $0{ }^{\circ} \mathrm{C}$ for $10 \mathrm{~min}$ before a solution 30 of $\quad r a c-(3 \mathrm{aS}, 4 \mathrm{a} R, 8 \mathrm{aS}, 9 \mathrm{a} S)-1,9-$ dioxododecahydronaphtho[2,3c]furan-4-carbaldehyde $(130 \mathrm{mg}, 0.55 \mathrm{mmol})$ in anhydrous 2MeTHF $(10 \mathrm{~mL})$ was added. The reaction mixture was stirred at 0 ${ }^{\circ} \mathrm{C}$ for a further $1 \mathrm{~h}$ and then quenched with sat. aq. $\mathrm{NH}_{4} \mathrm{Cl}(5$ $\mathrm{mL})$. An extraction into EtOAc $(10 \mathrm{~mL})$ was done and the 35 organic phase was washed with brine $(5 \mathrm{~mL})$, dried (hydrophobic frit) and concentrated in vacuo to yield a crude yellow gum. Purification by automated column chromatography (0-100\% EtOAc/cyclohexane) yielded $\left(3 \mathrm{a} S^{*}, 4 R^{*}, 4 \mathrm{a} R^{*}, 8 \mathrm{a} S^{*}, 9 \mathrm{a} S^{*}\right)-4-((E)-$ 2-(5-(3-(trifluoromethyl)phenyl)pyridin-2-

$40 \mathrm{yl})$ vinyl)octahydronaphtho[2,3-c]furan-1,9(3H,9aH)-dione $\quad(76.0$ $\mathrm{mg}, 0.17 \mathrm{mmol}, 25 \%)$ as a yellow glassy solid; ${ }^{1} \mathrm{H}$ NMR $(600$ $\left.\mathrm{MHz}, \mathrm{CDCl}_{3}\right) \delta 8.82(1 \mathrm{H}, \mathrm{d}, J=2.0, \mathrm{CHN}), 7.90(1 \mathrm{H}, \mathrm{dd}, J=8.1$, 2.4, $\mathrm{CHCCHN}), 7.83\left(1 \mathrm{H}, \mathrm{s}, \mathrm{CHCCF}_{3}\right), 7.78(1 \mathrm{H}, \mathrm{d}, J=7.6$, $\left.\mathrm{CHCCHCCF}_{3}\right), 7.69\left(1 \mathrm{H}, \mathrm{d}, J=7.8, \mathrm{CHCHCCF}_{3}\right), 7.63(1 \mathrm{H}, \mathrm{t}, J$ $\left.45=7.6, \mathrm{CHCHCCF}_{3}\right), 7.36(1 \mathrm{H}, \mathrm{dd}, J=8.2,0.6, \mathrm{CHCN}), 6.70(1 \mathrm{H}$, $\left.\mathrm{d}, J=15.4, \mathrm{C}^{4} \mathrm{HCHCHPyr}\right), 6.56(1 \mathrm{H}, \mathrm{dd}, J=15.4,9.4$, $\left.\mathrm{C}^{4} \mathrm{HCHCHPyr}\right), 4.37$ (1H, d, $\left.J=9.4, \mathrm{C}^{3} \mathrm{HH}\right), 4.20(1 \mathrm{H}, \mathrm{dd}, J=$ 9.4, 4.8, $\left.\mathrm{C}^{3} \mathrm{H} H\right), 3.58\left(1 \mathrm{H}, \mathrm{d}, J=7.4, \mathrm{C}^{9 \mathrm{a}} H\right), 2.85(1 \mathrm{H}, \mathrm{ddd}, J=$ $\left.11.1,7.4,4.8, \mathrm{C}^{3 \mathrm{a}} H\right), 2.34\left(1 \mathrm{H}, \mathrm{td}, J=11.1,9.4, \mathrm{C}^{4} H\right), 2.20-2.08$ ${ }_{50}\left(1 \mathrm{H}, \mathrm{m}, \mathrm{C}^{8 \mathrm{a}} \mathrm{H} \& \mathrm{C}^{8} \mathrm{HH}\right), 2.02-1.94\left(1 \mathrm{H}, \mathrm{m}, \mathrm{C}^{5} \mathrm{HH}\right), 1.86-1.66$ $\left(2 \mathrm{H}, \mathrm{m}, \mathrm{C}^{6} H \mathrm{H} \& \mathrm{C}^{7} H \mathrm{H}\right), 1.56\left(1 \mathrm{H}, \mathrm{qd}, J=11.2,3.4, \mathrm{C}^{4 \mathrm{a}} H\right), 1.34-$ $1.00\left(4 \mathrm{H}, \mathrm{m}, \mathrm{C}^{5} \mathrm{H} H, \mathrm{C}^{6} \mathrm{H} H, \quad \mathrm{C}^{7} \mathrm{H} H \& \mathrm{C}^{8} \mathrm{H} H\right) ;{ }^{13} \mathrm{C}$ NMR $(150$ $\left.\mathrm{MHz}, \mathrm{CDCl}_{3}\right) \delta 203.0\left(C^{9} \mathrm{O}\right), 172.1\left(C^{l} \mathrm{O}\right), 152.6(C \mathrm{~N}), 147.8$ $(C \mathrm{HN}), 138.2\left(\mathrm{CCHCCF}_{3}\right), 134.7(\mathrm{CHCCHN}), 134.4(\mathrm{CCHN})$, 55134.3 (C $\left.{ }^{4} \mathrm{HCHCHPyr}\right), 131.9$ (C $\left.{ }^{4} \mathrm{HCHCHPyr}\right), 131.8$ (q, $J=32.1$, $\left.\mathrm{CCF}_{3}\right), 130.3\left(\mathrm{CHCCHCCF}_{3}\right), 129.9\left(\mathrm{CHCHCCF}_{3}\right), 125.1$ (q, $J=$ 3.6, $\left.\mathrm{CHCHCCF}_{3}\right), 123.9$ (q, $\left.J=3.6, \mathrm{CCHCCF}_{3}\right), 124.0(\mathrm{~d}, J=$ 272.0, $\left.C \mathrm{~F}_{3}\right), 122.5(C \mathrm{HCN}), 70.5\left(C^{3} \mathrm{H}_{2}\right), 53.9\left(C^{9 a} \mathrm{H}\right), 52.0$
$\left(C^{8 a} \mathrm{H}\right), 48.2\left(C^{4} \mathrm{H}\right), 44.5\left(C^{3 a} \mathrm{H}\right), 44.4\left(C^{4 a} \mathrm{H}\right), 32.7\left(C^{5} \mathrm{H}_{2}\right), 25.4$ ${ }_{60}\left(C^{8} \mathrm{H}_{2}\right), 25.3\left(C^{6} \mathrm{H}_{2}\right), 24.9\left(C^{7} \mathrm{H}_{2}\right)$; IR (thin film) $2930(\mathrm{C}-\mathrm{H})$, $2858(\mathrm{C}-\mathrm{H}), 1772(\mathrm{C}=\mathrm{O}), 1706(\mathrm{C}=\mathrm{O}), 1335,1167,1119,1072$, 998, 971, $810 \mathrm{~cm}^{-1} ; \mathrm{m} / \mathrm{z}(\mathrm{ES}+) 455\left(100 \%,[\mathrm{M}+\mathrm{H}]^{+}\right)$; HRMS (EI) calcd for $\mathrm{C}_{26} \mathrm{H}_{24} \mathrm{O}_{3} \mathrm{~F}_{3} \mathrm{~N}[\mathrm{M}+\mathrm{H}]^{+} 455.17028$, observed 455.17029. Chiral column purification $(70 \%$ EtOH/Heptane, $\mathrm{f}=20 \mathrm{ml} / \mathrm{min}$, ${ }_{65}$ Column $30 \mathrm{~mm} \quad \mathrm{x} \quad 25 \mathrm{~cm}$ Chiralpak AD-H) gave (+)- $(3 \mathrm{a} S, 4 R, 4 \mathrm{a} R, 8 \mathrm{a} S, 9 \mathrm{a} S)-4-((E)-2-(5-(3-$

(trifluoromethyl)phenyl)pyridin-2-

yl)vinyl)octahydronaphtho[2,3-c]furan-1,9(3H,9aH)-dione

$((+)-16)$ with an $[\alpha]_{\mathrm{D}}^{25}=+31.4 \quad(\mathrm{MeOH})$ and

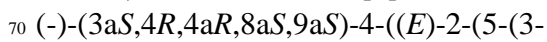

(trifluoromethyl)phenyl)pyridin-2-

yl)vinyl)octahydronaphtho[2,3-c]furan-1,9(3H,9aH)-dione $((-)-16)$ with an $[\alpha]_{\mathrm{D}}^{25}=-31.3(\mathrm{MeOH})$.

\section{Notes and references}

$75{ }^{a}$ Department of Chemistry, University College London, 20 Gordon Street, London,WC1H 0AJ, UK. E-mails: v.chudasama@ucl.ac.uk and s.caddick@ucl.ac.uk.

${ }^{b}$ Centre for Inflammation and Tissue Repair, 5 University Street, London WC1E 6JJ.

${ }_{80}{ }^{c}$ GSK, Gunnels Wood Road, Stevenage, Herts SG1 $2 N Y$.

$\dagger$ Electronic Supplementary Information (ESI) available: $\left[{ }^{1} \mathrm{H}\right.$ and ${ }^{13} \mathrm{C}$ NMR spectra of all novel compounds]. See DOI: 10.1039/b000000x/

\$ Without any information on the absolute stereochemistry of either enantiomer of any one of 14-16 it is not appropriate to postulate as to 85 what is the precise structure of each enantiomer.

1 M. J. Krantz and S. Kaul, JAMA Internal Medicine, 2015, 175, 9-10.

2 P. F. Mercer and R. C. Chambers, Biochim Biophys Acta., 2013, 1832, 1018-1027.

903 M. Casey and R. McCarthy, Synlett, 2011, 06, 801-804.

4 M. Yamaguchi, M. Tsukamoto and I. Hirao, Tetrahedron Lett., 1985, 26, 1723-1726.

5 C. Harcken, R. Brückner and E. Rank, Chem. Eur. J., 1998, 4, 2342-2352.

${ }_{95} 6$ Y. Xia, S. Chackalamannil, M. Clasby, D. Doller, K. Eagen, W. J. Greenlee, H. G. Tsai, J. Agans-Fantuzzi, H. S. Ahn, G. C. Boykow, Y. S. Hsieh, C. A. Lunn and M. Chintala, Bioorg. Med. Chem. Lett., 2007, 17, 4509-4513.

7 T. Fukuyama, S. C. Lin and L. Li, J. Am. Chem. Soc., 1990, 112, 7050-7051.

8 M. C. Clasby, S. Chackalamannil, M. Czarniecki, D. Doller, K. Eagen, W. Greenlee, G. Kao, Y. Lin, H. Tsai, Y. Xia, H. S. Ahn, J. Agans-Fantuzzi, G. Boykow, M. Chintala, C. Foster, A. Smith-Torhan, K. Alton, M.

105 Bryant, Y. Hsieh, J. Lau and J. Palamanda, J. Med. Chem., 2007, 50, 129-138.

9 S. Chackalamannil, Y. Wang, W. J. Greenlee, Z. Hu, Y. Xia, H. S. Ahn, G. Boykow, Y. Hsieh, J. Palamanda, J. Agans-Fantuzzi, S. Kurowski, M. Graziano, M. Chintala, $110 \quad$ J.Med. Chem., 2008, 51, 3061-3064.

10 Y. Xia, S. Chackalamannil, T. M. Chan, M. Czarniecki, D. Doller, K. Eagen, W. Greenlee, H. Tsai, Y. Wang, H. S. Ahn, G. Boykow, A. T. McPhail, Bioorg. Med. Chem. Lett., 2006, 16, 4969-4972

11511 S. Chackalamannil, Y. Xia, W. J. Greenlee, M. Clasby, D. Doller, H. Tsai, T. Asberom, M. Czarniecki, H. S. Ahn, G. Boykow, C. Foster, J. Agans-Fantuzzi, M. Bryant, J. Lau and M. Chintala, J. Med. Chem., 2005, 48, 5884-5887.

12 (a) K. S. Schroeder and B. D. Neagle, J. Biomol. Screen., 1996, 1, 75-80; (b) A. Ortiz-Stern, X. Deng, N.

Smoktunowicz, P. F. Mercer and R. C. Chambers, J. Cell. Physiol., 2012, 227, 3575-3584. 
13 B. P. Damiano, C. K. Derian, B. E. Maryanoff, H. C. Zhang and P. A. Gordon, Cardiovasc. Drug Rev., 2003, 21, 313-326.

14 M. V. Chelliah, K. Eagen, Z. Guo, S. Chackalamannil, Y. Xia, H. Tsai, W. J. Greenlee, H.-S. Ahn, S. Kurowski, G. Boykow, Y. Hsieh and M. Chintala, ACS Med. Chem. Lett., 2014, 5, 561-565 and references therein. 